\title{
Article \\ Application of Induced Preorderings in Score Function-Based Method for Solving Decision-Making with Interval-Valued Fuzzy Soft Information
}

\author{
Mabruka Ali ${ }^{1}$, Adem Kiliçman ${ }^{1,2, *(\mathbb{D})}$ and Azadeh Zahedi Khameneh ${ }^{2}$ (D) \\ 1 Department of Mathematics, Universiti Putra Malaysia, Serdang UPM 43400, Malaysia; \\ altwer2016@gmail.com \\ 2 Institute for Mathematical Research, Universiti Putra Malaysia, Serdang UPM 43400, Malaysia; \\ zk.azadeh@upm.edu.my \\ * Correspondence:akilic@upm.edu.my
}

check for updates

Citation: Ali, M.; Kılıçman, A.; Zahedi Khameneh, A. Application of Induced Preorderings in Score

Function-Based Method for Solving Decision-Making with Interval-Valued Fuzzy Soft Information. Mathematics 2021, 9 , 1575. https://doi.org/10.3390/math 9131575

Academic Editor: Sorin Nadaban

Received: 18 May 2021

Accepted: 29 June 2021

Published: 4 July 2021

Publisher's Note: MDPI stays neutral with regard to jurisdictional claims in published maps and institutional affiliations.

Copyright: (c) 2021 by the authors. Licensee MDPI, Basel, Switzerland. This article is an open access article distributed under the terms and conditions of the Creative Commons Attribution (CC BY) license (https:// creativecommons.org/licenses/by/ $4.0 /)$.

\begin{abstract}
Ranking interval-valued fuzzy soft sets is an increasingly important research issue in decision making, and provides support for decision makers in order to select the optimal alternative under an uncertain environment. Currently, there are three interval-valued fuzzy soft set-based decision-making algorithms in the literature. However, these algorithms are not able to overcome the issue of comparable alternatives and, in fact, might be ignored due to the lack of a comprehensive priority approach. In order to provide a partial solution to this problem, we present a group decisionmaking solution which is based on a preference relationship of interval-valued fuzzy soft information. Further, corresponding to each parameter, two crisp topological spaces, namely, lower topology and upper topology, are introduced based on the interval-valued fuzzy soft topology. Then, using the preorder relation on a topological space, a score function-based ranking system is also defined to design an adjustable multi-steps algorithm. Finally, some illustrative examples are given to compare the effectiveness of the present approach with some existing methods.
\end{abstract}

Keywords: interval-valued fuzzy soft sets; interval-valued fuzzy soft topology; preference relationship; decision-making

\section{Introduction}

Dealing with vagueness and uncertainty, rather than exactness, in most real-world situations is the main problem in data-analysis sciences and decision-making. Many mathematical theories and tools such as probability theory, fuzzy set theory [1], interval-valued fuzzy set theory [2], intuitionist fuzzy set theory [3], rough set theory [4] and soft set theory [5] have been implemented to handle this problem, with the latter allowing researchers to deal with parametric data. Nowadays, soft sets theory contributes to a vast range of applications, particularly in decision-making. In this regard, many important results have been achieved, from parameter reduction to new ranking models.

Many soft set extensions and their applications have been discussed in previous studies, such as fuzzy soft sets [6-13] intuitionistic fuzzy sets [14-17], rough soft sets [18,19] and fuzzy soft topology [20-23]. The interval-valued fuzzy soft method was first used for decision-making problems by Son [24]. He applied this method by using the comparison table. Yang et al. [25] developed the method presented in [7] for an interval-valued fuzzy soft set and then, applied the concept of interval-valued fuzzy choice values to propose an approach for solving decision-making problems. The notion of level set in decisionmaking based on interval-valued fuzzy soft sets was introduced by Feng et al. [26] and then, the level soft set for interval-valued fuzzy soft sets was developed, further see [27]. Khameneh et al. [28-30] introduced the preference relationship for both fuzzy soft sets and intuitionistic fuzzy soft sets and then selected an optimal option for group decision-making problems by defining a new function value. In addition, interval-valued fuzzy soft sets have also been applied to various fields, for example information measure [31-34], decision making [35-38], matrix theory [39-41], and parameter reduction [37,38,42]. 
Recently, Ma et al. [43] introduced an average and an antitheses table for intervalvalued fuzzy soft sets and then selected an optimal option for group decision-making problems through the score value. Ma et al. [44] developed two methods [26,45] to solve decision-making problems by providing a new efficient decision-making algorithm and also considering added objects. However, these methods did not address the problem of incomparable alternatives because they lack a comprehensive priority approach. In order to solve these issues, this paper proposes an application of the induced preorderings based method for solving decision-making with interval-valued fuzzy soft information.Our contributions are as follows:

1 Proposing application of induced preordering based method for solving decisionmaking with interval-valued fuzzy soft information.

2 Proposing a novel score function of interval-valued fuzzy soft sets that selects an optimal option for group decision-making problems.

3 A real-life example is given to compare the effectiveness of this approach with some existing methods.

\section{Preliminaries}

In this section, we recall some definitions and properties of interval-valued fuzzy sets $(I V F)$ and interval-valued fuzzy soft sets (IVFS). Note that, throughout this paper, $X$ and $E$ denote the sets of objects and parameters, respectively. $\mathbb{I}^{X}$ and $[\mathbb{I}]^{X}$, where $\mathbb{I}=[0,1]$ and $[\mathbb{I}]=\{[a, b], a \leq b, a, b \in \mathbb{I}\}$ denote, respectively, the set of all fuzzy subsets and the set of all interval-valued fuzzy subsets of $X$.

Definition 1. Ref. [2] A pair $(f, X)$, is called an IVF subset of $X$ if $f$ is a mapping given by $f: X \rightarrow[\mathbb{I}]$ such that for any $x \in X, f(x)=\left[f^{-}(x), f^{+}(x)\right]$ is a closed subinterval of $[0,1]$ where $f^{-}(x)$ and $f^{+}(x)$ are referred to as the lower and upper degrees of membership $x$ to $f$ and $0 \leq f^{-}(x) \leq f^{+}(x) \leq 1$.

In 1999, Molodtsov [5] defined the concept of soft sets (SS) for the first time as a pair of $(f, E)$ or $f_{E}$ such that $E$ is a parameter set and $f$ is the mapping $f: E \rightarrow 2^{X}$ where for any $e \in E, f(e)$ is a subset of $X$. By combining the concepts of soft sets and interval-valued fuzzy sets, a new hybrid tool was defined as the following.

Definition 2. Ref. [25] A pair $(f, E)$ is called an IVFS set over X if the mapping $f$ is given by $f: E \rightarrow[\mathbb{I}]^{X}$ where for any $e \in E$ and $x \in X, f(e)(x)=\left[f^{-}(e)(x), f^{+}(e)(x)\right]$.

Consider two IVFSs $f_{E}, g_{E}$ over the common universe $X$. The union of $f_{E}$ and $g_{E}$, denoted by $f_{E} \tilde{\nabla} g_{E}$, is the IVFSS $(f \tilde{\nabla} g)_{E}$, where $\forall e \in E$ and any $x \in X$, we have $(f \tilde{\nabla} g)(e)(x)=\left[\max \left\{f_{e}^{-}(x), g_{e}^{-}(x)\right\}, \max \left\{f_{e}^{+}(x), g_{e}^{+}(x)\right\}\right]$. The intersection of $f_{E}$ and $g_{E}$, denoted by $f_{E} \tilde{\wedge} g_{E}$, is the IVFSs $(f \tilde{\wedge} g)_{E}$, where $\forall e \in E$ and $\forall x \in X$, we have $(f \tilde{\wedge} g)(e)(x)=\left[\min \left\{f_{e}^{-}(x), g_{e}^{-}(x)\right\}, \min \left\{f_{e}^{+}(x), g_{e}^{+}(x)\right\}\right]$. The complement of $f_{E}$ is denoted by $f_{E}^{c}$ and is defined by $f^{c}: E \rightarrow[\mathbb{I}]^{X}$ where $\forall e \in E$ and any $x \in X, f^{c}(e)(x)=$ $\left[1-f_{e}^{+}(x), 1-f_{e}^{-}(x)\right]$. The null IVFSs, denoted by $\varnothing_{E}$, is defined as an IVFSs over $X$ such that $f_{e}^{-}(x)=f_{e}^{+}(x)=0$ for all $e \in E$ and any $x \in X$. The absolute IVFSs, denoted by $X_{E}$, is defined as an IVFSs over $X$ where $f_{e}^{-}(x)=f_{e}^{+}(x)=1, \forall e \in E$ and any $x \in X$.

Using the matrix form of interval-valued fuzzy relations, authors in [39] represented a finite IVFSs $f_{E}$ as the following $n \times m$ matrix

$$
f_{E}=\left[\left[f_{i j}^{-}, f_{i j}^{+}\right]\right]_{n \times m}=\left[\begin{array}{ccc}
{\left[f_{e_{1}}^{-}\left(x_{1}\right), f_{e_{1}}^{+}\left(x_{1}\right)\right]} & \ldots & {\left[f_{e_{1}}^{-}\left(x_{m}\right), f_{e_{1}}^{+}\left(x_{m}\right)\right]} \\
\vdots & \ldots & \vdots \\
{\left[f_{e_{n}}^{-}\left(x_{1}\right), f_{e_{1}}^{+}\left(x_{1}\right)\right]} & \ldots & {\left[f_{e_{n}}^{-}\left(x_{m}\right), f_{e_{1}}^{+}\left(x_{m}\right)\right]}
\end{array}\right]_{n \times m}
$$

where $|E|=n,|X|=m$ and $f_{i j}^{-}=f_{e_{i}}^{-}\left(x_{j}\right), f_{i j}^{+}=f_{e_{i}}^{+}\left(x_{j}\right)$ for $i=1, \ldots, n$ and $j=1, \ldots, m$.

Accordingly, the concepts of union, intersection, complement, etc., can be represented in a matrix format in the finite case. 
Definition 3. Ref. [46] A triplet $(X, E, \tau)$ is called an interval-valued fuzzy soft topological space (IVFST) if $\tau$ is a collection of interval-valued fuzzy soft subsets of X containing absolute and null IVFSs and closed under arbitrary union and finite intersection.

Preorders and Topologies

In this subsection, we present some basic properties about the connection between preorders and topologies proposed by [47].

Topological structures and classical order structures are well recognised to have close relationships, which can be summarised as follows:

(1) A subset $A$ of $X$ is called an upper set of $X$ if $A=\uparrow A$, where $\uparrow A$ defined by $\uparrow A=\{y \in X: \exists x \in A, x \leq y\}$, and $X$ is a preordered set, and $B$ is called a lower set $B=\downarrow B=\{y \in X: \exists x \in X, y \leq x\}$.

(2) The family of all upper subsets of $x$ is a topology for a preorder set $(X, \leq)$, which is called the Alexandrov topology induced in $(X, \leq)$.

(3) A topological space $(X, \tau)$ is defined by $x \leq y$ if and only if $x \in U$, then $y \in U$ for each open set $U$ of $X$, or equivalently $x \in c\{y\}$, where $c\{y\}$, is the closure of $\{y\}$. Then, $\leq$ is a preorder on $X$, called the specialization order $(X, \tau)$ on $X$.

\section{Construction Tow Preorderings in Lower and Upper Spaces}

By using the notion of $\left[\alpha_{1}, \alpha_{2}\right]$-level sets of interval-valued fuzzy soft open sets in $(X, E, \tau)$, this section, introduces two topological spaces, known as lower and upper spaces, by which two preordering relations over the universal set $X$ are investigated.

Definition 4. Let $f_{E}$ be an IVFS set over X. Corresponding to each parameter $e \in E$, we define two crisp sets, called $\alpha$-upper-e crisp set and $\beta$-lower-e crisp set, where $\alpha=\left[\alpha_{1}, \alpha_{2}\right] \subset \mathbb{I}, \beta=$ $\left[\beta_{1}, \beta_{2}\right] \subset \mathbb{I}$ as the following:

$$
\begin{aligned}
\text { U.C.S } f_{\alpha}^{f}(e) & =\left\{x \in X:\left[f_{e}^{-}(x), f_{e}^{+}(x)\right]>\alpha, \alpha \subseteq[0,1)\right\} \\
& =\left\{x \in X: f_{e}^{-}(x)>\alpha_{1}, f_{e}^{+}(x)>\alpha_{2}, \alpha_{1}, \alpha_{2} \in[0,1)\right\} \\
\text { L.C.S } f_{\beta}(e) & =\left\{x \in X:\left[f_{e}^{-}(x), f_{e}^{+}(x)\right]<\beta, \beta \subseteq(0,1]\right\} \\
& =\left\{x \in X: f_{e}^{-}(x)<\beta_{1}, f_{e}^{+}(x)<\beta_{2}, \beta_{1}, \beta_{2} \in(0,1]\right\}
\end{aligned}
$$

Proposition 1. Let $X$ be the set of objects, $E$ be the set of parameters and $f_{E}, g_{E}$ be two IVFSs over $X$. Suppose that the threshold intervals $\alpha_{1}, \alpha_{2}, \subseteq[0,1)$, and $\beta_{1}, \beta_{2}, \subseteq(0,1]$ are given such that $\alpha_{1}=\left[\alpha_{1}^{\star}, \alpha_{1}^{\star \star}\right], \alpha_{2}=\left[\alpha_{2}^{\star}, \alpha_{2}^{\star \star}\right], \beta_{1}=\left[\beta_{1}^{\star}, \beta_{1}^{\star \star}\right]$ and $\beta_{2}=\left[\beta_{2}^{\star}, \beta_{2}^{\star \star}\right]$. Consider the parameter $e \in E$.

1. If $\alpha_{1} \geq \alpha_{2}$, then U.C. $S_{\alpha_{1}}^{f}(e) \subseteq$ U.C. $S_{\alpha_{2}}^{f}(e)$. If $\beta_{1} \geq \beta_{2}$, then L.C.S $\beta_{\beta_{2}}^{f}(e) \subseteq$ L.C.S $\beta_{\beta_{1}}^{f}(e)$.

2. If $f_{E} \tilde{\leq} g_{E}$, then U.CS $S_{\alpha_{1}}^{f}(e) \subseteq U . C . S_{\alpha_{1}}^{g}(e)$ and L.C. $S_{\beta_{1}}^{f}(e) \subseteq$ L.C. $S_{\beta_{1}}^{g}(e)$.

3. If $f_{E}=X$, then U.C. $S_{\alpha_{1}}^{f}(e)=X$ and L.C. $S_{\beta_{1}}^{f}(e)=\varnothing$. Moreover, if $f_{E}=\varnothing_{E}$ then, U.CS $S_{\alpha_{1}}^{f}(e)=\varnothing$ and L.CS $S_{\beta_{1}}^{f}(e)=X$.

4. U.C. $S_{\alpha_{1}}^{f}(\neg e)=\operatorname{L} \cdot C S_{\left[1-\alpha_{1}^{\star \star}, 1-\alpha_{1}^{\star}\right]}^{f}(e)$ and $\operatorname{L.CS}_{\alpha_{1}}^{f}(\neg e)=U \cdot C S_{\left[1-\alpha_{1}^{\star \star}, 1-\alpha_{1}^{\star}\right]}^{f}(e)$.

5. $\quad U . C S_{\alpha_{1}}^{\neg f}(e)=\operatorname{L} \cdot C S_{\left[1-\alpha_{1}^{\star \star}, 1-\alpha_{1}^{\star}\right]}^{f}(e)$ and $\operatorname{L.CS}_{\alpha_{1}}^{\neg f}(\neg e)=U \cdot \operatorname{Des}_{\left[1-\alpha_{1}^{\star \star}, 1-\alpha_{1}^{\star}\right]}^{f}(e)$.

Proof. It is straightforward.

Theorem 1. Let $(X, E, \tau)$ be an IVFSTS. Suppose that the threshold intervals $\alpha_{1}, \alpha_{2}, \subseteq[0,1)$, and $\beta_{1}, \beta_{2}, \subseteq(0,1]$ are given such that $\alpha=\left[\alpha_{1}, \alpha_{2}\right]$ and $\beta=\left[\beta_{1}, \beta_{2}\right]$, then

1. The collection $\left\{\right.$ U.C. $\left.S_{\alpha}^{f}(e): f_{E} \in \tau, e \in E, \alpha \subseteq[0,1)\right\}$, denoted by $\tau_{e, \alpha}^{u}$, is a topology over X.

2. The collection $\mathfrak{B}_{\beta}^{l}(e)=\left\{\right.$ L.C. $\left.S_{\beta}^{f}(e): f_{E} \in \tau, e \in E, \beta \subseteq(0,1]\right\}$, is a base for a topology over $X$, denoted by $\tau_{e, \beta}^{l}$. 
Proof. 1. (a) By Proposition $1, X, \varnothing \in \tau_{e, \alpha}^{u}$, since $X_{E}, \varnothing_{E} \in \tau$.

(b) Let $\left\{\text { U.C.S. } S_{\alpha}^{f_{i}}(e)\right\}_{i \in I}$ be a subfamily of $\tau_{e, \alpha}^{u}$. Then, we have $\bigcup_{i} U . C . S_{\alpha}^{f_{i}}(e)$ $=U . C . S_{\alpha}^{\left(\tilde{\nabla}_{i \in I} f_{i}\right)}(e) \in \tau_{e, \alpha}^{u}$, since $\tilde{\nabla}_{i \in I} f_{i E} \in \tau$.

(c) Let U.C.S. $S_{\alpha}^{f}(e)$ and U.C.S. $S_{\alpha}^{g}(e)$ be two open sets in $\tau_{e, \alpha}^{u}$. Then, we have $U . C S_{\alpha}^{f}(e) \cap$ U.C. $S_{\alpha}^{g}(e)=U . C . S_{\alpha}^{(f \tilde{\wedge} g)}(e) \in \tau_{e, \alpha}^{u}$, since $f_{E} \tilde{\wedge} g_{E} \in \tau$. This completes the proof.

2. (a) That $X \in \mathfrak{B}_{\beta}^{l}(e)$ is implied from $\varnothing_{E}$ is in $\tau$.

(b) Let L.C.S $S_{\beta}^{f}(e)$ and L.C.S $S_{\beta}^{g}(e)$ in $\mathfrak{B}_{\beta}^{l}(e)$. Then, we have L.C.S $f_{\beta}^{f}(e) \cap L . C . S_{\beta}^{g}(e)=$ L.C. $S_{\beta}^{f \tilde{g} g}(e) \in \mathfrak{B}_{\beta}^{l}(e)$ that is implied form $f \tilde{\nabla} g \in \tau$.

Theorem 2. Let $(X, E, \tau)$ be an IVFSTS. Suppose that the threshold intervals $\alpha_{1}, \alpha_{2}, \subseteq[0,1)$, and $\beta_{1}, \beta_{2}, \subseteq(0,1]$ are given such that $\alpha=\left[\alpha_{1}, \alpha_{2}\right]$ and $\beta=\left[\beta_{1}, \beta_{2}\right]$.

1. The binary relation $\succsim_{e, \alpha}^{\tau}$ on $X$ defined by

$$
y \succeq_{e, \alpha}^{\tau} x \Leftrightarrow\left[\forall V \in \tau_{e, \alpha}^{u}: x \in V \Rightarrow y \in V\right]
$$

is a preorder relation called $\alpha$-upper-e preorder relation on $X$.

2. The binary relation $\preceq_{e}^{\tau, \beta}$ on $X$ defined by

$$
y \preceq_{e}^{\tau, \beta} x \Leftrightarrow\left[\forall U \in \tau_{e, \beta}^{l}: x \in U \Rightarrow y \in U\right]
$$

is a preorder relation called $\beta$-lower-e preorder relation on $X$.

Proof. 1. For all $x \in X$, obviously, $x \succsim_{e, \alpha}^{u} x$, that is, " $\succsim_{e, \alpha}^{u}$ " is reflexive. Now, for all $x, y, z \in X$, if $y \succsim_{e, \alpha}^{u} x$, and $z \succsim_{e, \alpha}^{u} y$, then, if for all $V \in \tau_{e, \alpha}^{u}$-open set, $x \in V$, then $y \in V$ and $z \in V$, so, $z \succsim_{e, \alpha}^{u} x$, that is, " $\succsim_{e, \alpha}^{u}$ " is transitive. Thererfore, $\left(X, \succsim_{e, \alpha}^{u}\right)$ is a preordered set.

2. A similar technique is used to prove the second part.

Theorem 3. Let $(X, E, \tau)$ be an IVFSTS. Suppose that the threshold intervals $\alpha_{1}, \alpha_{2}, \subseteq[0,1)$, and $\beta_{1}, \beta_{2}, \subseteq(0,1]$ are given such that $\alpha=\left[\alpha_{1}, \alpha_{2}\right]$ and $\beta=\left[\beta_{1}, \beta_{2}\right]$.

1. The binary relation $\simeq_{e, \alpha}^{\tau}$, defined by

$$
y \simeq_{e, \alpha}^{\tau} x \Leftrightarrow\left[y \succsim_{e, \alpha}^{\tau} x, x \succsim_{e, \alpha}^{\tau} y\right]
$$

is an equivalence relation over $X$. If $y \simeq_{e, \alpha}^{\tau} x$, then we say $x$ and $y$ are $\alpha$-upper equivalent with to respect to the parameter $e$.

The equivalence relation $\simeq_{e, \alpha}^{\tau}$, generates the partition $P_{e, \alpha}^{\tau}$ of $X$ where the equivalence classes are defined as $[x]_{e, \alpha}^{\tau}=\left\{z \in X: z \simeq_{e, \alpha}^{\tau} x\right.$ and are called $\alpha$-upper-e equivalence classes.

2. The binary relation $\simeq_{e}^{\tau, \beta}$, where $\beta=\left[\beta_{1}, \beta_{2}\right]$,

$$
y \simeq_{e}^{\tau, \beta} x \Leftrightarrow\left[y \precsim_{e^{\tau, \beta}} x, x \precsim_{e^{\tau, \beta}} y\right]
$$

is an equivalence relation over $X$. If $y \simeq_{e, \beta}^{\tau} x$, then we say $x$ and $y$ are $\left[\beta_{1}, \beta_{2}\right]$-lower equivalent with to respect to the parameter $e$. The equivalence relation $\simeq_{e}^{\tau, \beta}$, generates the partition $P_{e}^{\tau, \beta}$ of $X$ where the equivalence classes are defined as $[x]_{e}^{\tau, \beta}=\left\{z \in X: z \simeq_{e}^{\tau, \beta}\right.$ x and are called $\beta$-lower-e equivalence classes.

Proof. It is straightforward.

Preorder and Equivalence Matrices

Now, let the finite sets $X=\left\{x_{1}, \cdots, x_{m}\right\}$ and $E=\left\{e_{1}, \cdots, e_{n}\right\}$ be given as the sets of objects and parameters. Then, the previous properties can be represented by using the matrix form of IVFS sets as the following. 
Take an IVFS set $f_{E}$ over X. First, for any $1 \leq i \leq m$ and $1 \leq t \leq n$, the concepts of $\alpha$-upper- $e_{t}$ and $\beta$-lower- $e_{t}$ matrices of $f_{E}$, where $\alpha, \beta \subseteq \mathbb{I}$, can be formulated as the following two matrices (or row vectors)

$$
\text { U.C. } S_{\alpha} e_{t}^{f}=\left[u_{i}^{f}\left(e_{t}, \alpha\right)\right]_{1 \times m}= \begin{cases}1 & \text { if } f_{e_{t}}^{-}\left(x_{i}\right)>\alpha_{1}, f_{e_{t}}^{+}\left(x_{i}\right)>\alpha_{2} \\ 0 & \text { if } f_{e_{t}}^{-}\left(x_{i}\right) \leq \alpha_{1}, f_{e_{t}}^{+}\left(x_{i}\right) \leq \alpha_{2}\end{cases}
$$

and

$$
\text { L.C.S. } S_{\beta} e_{t}^{f}=\left[l_{i}^{f}\left(e_{t}, \beta\right)\right]_{1 \times m}= \begin{cases}0 & \text { if } f_{e_{t}}^{-}\left(x_{i}\right) \geq \beta_{1}, f_{e_{t}}^{+}\left(x_{i}\right) \geq \beta_{2} \\ 1 & \text { if } f_{e_{t}}^{-}\left(x_{i}\right)<\beta_{1}, f_{e_{t}}^{+}\left(x_{i}\right)<\beta_{2}\end{cases}
$$

where $\alpha=\left[\alpha_{1}, \alpha_{1}\right]$ and $\beta=\left[\beta_{1}, \beta_{2}\right]$ are the given threshold vectors.

Then, obviously, for any $e_{t} \in E$, the topologies $\tau_{e_{t}, \alpha}^{u}$ and $\tau_{e_{t}, \beta}^{l}$ can be represented by the collections

$$
\tau_{e_{t}, \alpha}^{u}=\left\{\left[u_{i}^{f}\left(e_{t}, \alpha\right)\right]_{1 \times m}: \alpha \subseteq[0,1), f_{E} \in \tau, 1 \leq i \leq m\right\}
$$

and

$$
\tau_{e_{t}, \beta}^{l}=\left\{\left[l_{i}^{f}\left(e_{t}, \beta\right)\right]_{1 \times m}: \beta \subseteq(0,1], f_{E} \in \tau, 1 \leq i \leq m\right\}
$$

where $\tau$ is the IVFST on $X$.

Accordingly, the preorderings $\succeq_{e_{t}, \alpha}^{\tau}$ and $\preceq_{e_{t}}^{\tau, \beta}$ can be represented by

$$
x_{i} \succeq_{e_{t}, \alpha}^{\tau} x_{j} \Leftrightarrow\left[\forall f_{E} \in \tau: u_{j}^{f}\left(e_{t}, \alpha\right)=1 \Rightarrow u_{i}^{f}\left(e_{t}, \alpha\right)=1\right]
$$

and

$$
x_{i} \preceq_{e_{t}}^{\tau, \beta} x_{j} \Leftrightarrow\left[\forall f_{E} \in \tau: l_{j}^{f}\left(e_{t}, \beta\right)=1 \Rightarrow l_{i}^{f}\left(e_{t}, \beta\right)=1\right]
$$

where $x_{i}, x_{j} \in X$.

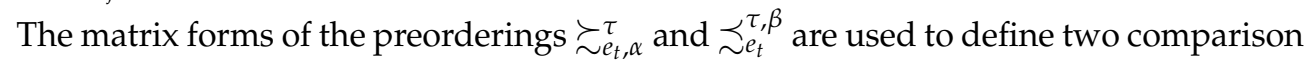
matrices $G_{\alpha}\left(e_{t}\right)=\left[g_{\alpha}\left(e_{t}\right)_{i j}\right]_{m \times m}$ and $S_{\beta}\left(e_{t}\right) \stackrel{\tau_{t}, \alpha}{=}\left[s_{\beta}\left(e_{t}\right)_{i j}\right]_{m \times m}$, which are two square matrices whose rows and columns are labeled by the objects of $X$, as below.

Definition 5. Consider the binary relations $\succsim_{e_{t}, \alpha}^{\tau}$ and $\precsim_{e_{t}}^{\tau, \beta}$ and threshold intervals $\alpha=\left[\alpha_{1}, \alpha_{2}\right]$, $\beta=\left[\beta_{1}, \beta_{2} \subseteq \mathbb{I}\right.$. Then, we define

$$
G_{\alpha}\left(e_{t}\right)=\left[g_{\alpha}\left(e_{t}\right)_{i j}\right]_{m \times m}: g_{\left[\alpha_{1}, \alpha_{2}\right]}\left(e_{t}\right)_{i j}=\left\{\begin{array}{cc}
1 & \text { if } x_{i} \succsim \tau e_{t, \alpha} x_{j} \\
0 & \text { otherwis }
\end{array}\right.
$$

and

$$
S_{\beta}\left(e_{t}\right)=\left[s_{\beta}\left(e_{t}\right)_{i j}\right]_{m \times m}: s_{\left[\beta_{1}, \beta_{2}\right]}\left(e_{t}\right)_{i j}=\left\{\begin{array}{cc}
1 & \text { if } x_{i} \precsim \precsim_{e}^{\tau, \beta} x_{j} \\
0 & \text { otherwis }
\end{array}\right.
$$

Proposition 2. Let $(X, E, \tau)$ be an IVFST and $G_{\alpha}(e)$ and $S_{\beta}(e)$ be two matrices defined in Equations (3) and (4). Then,

1. For $1 \leq i \leq m, g_{\alpha}\left(e_{t}\right)_{i i}=1$ and $s_{\beta}\left(e_{t}\right)_{i i}=1$,

2. If $g_{\alpha}\left(e_{t}\right)_{i j}=g_{\alpha}\left(e_{t}\right)_{j k}=1$, then $g_{\alpha}\left(e_{t}\right)_{i k}=1$. If $s_{\beta}\left(e_{t}\right)_{i j}=s_{\beta}\left(e_{t}\right)_{j k}=1$, then $s_{\beta}\left(e_{t}\right)_{i k}=1$.

3. $G_{\alpha}\left(e_{t}\right)$ and $S_{\beta}\left(e_{t}\right)$ are symmetric matrices.

where $i, j, k \in\{1, \ldots, m\}$

Proof. It is straightforward.

Proposition 3. Let $(X, E, \tau)$ be an IVFSTS and $\alpha, \beta \subseteq \mathbb{I}$, where $\alpha=\left[\alpha_{1}, \alpha_{1}\right]$ and $\beta=\left[\beta_{1}, \beta_{2}\right]$ are the threshold intervals, then

1. $G_{\alpha}\left(e_{t}\right)$ is an identity matrix if and only if $\neg\left(x_{i} \succeq_{e_{t} \alpha}^{\tau} x_{j}\right), \forall i, j=1, \ldots, m$ and $i \neq j$.

2. $S_{\beta}\left(e_{t}\right)$ is an identity matrix if and only if $\neg\left(x_{i} \preceq_{e_{t}, \beta}^{\tau,} x_{j}\right), \forall i, j=1, \ldots, m$ and $i \neq j$.

3. $G_{\alpha}\left(e_{t}\right)$ is a unit matrix if and only if $x_{i} \succeq_{e_{t}, \alpha}^{\tau} x_{j}, \forall i, j=1, \ldots, m$ and $i \neq j$.

4. $S_{\beta}\left(e_{t}\right)$ is a unit matrix if and only if $x_{i} \preceq_{e_{t}}^{\tau, \beta} x_{j}, \forall i, j=1, \ldots, m$ and $i \neq j$. 
Proof. It is straightforward.

Proposition 4. Let $(X, E, \tau)$ be an IVFSTS and $\alpha, \beta \subseteq \mathbb{I}$, where $\alpha=\left[\alpha_{1}, \alpha_{1}\right], \beta=\left[\beta_{1}, \beta_{2}\right]$ are the threshold intervals, then

1. $G_{\alpha}\left(e_{t}\right)=I_{m}^{U}$ if and only if we have $x_{1} \succeq_{e_{t}, \alpha}^{\tau} \cdots \succeq_{e_{t}, \alpha}^{\tau} x_{m}$.

2. $S_{\beta}\left(e_{t}\right)=I_{m}^{U}$ if and only if $x_{1} \preceq_{e_{t}}^{\tau, \beta} \cdots \preceq_{e_{t}}^{\tau, \beta} x_{m}$.

3. $G_{\alpha}\left(e_{t}\right)=I_{m}^{L}$ if and only if $x_{m} \succeq_{e_{t}, \alpha}^{\tau} \cdots \succeq_{e_{t}, \alpha}^{\tau} x_{1}$.

4. $\quad S_{\beta}\left(e_{t}\right)=I_{m}^{L}$ if and only if $x_{m} \preceq_{e_{t}}^{\tau, \beta} \ldots \preceq_{e_{t}}^{\tau, \beta} x_{1}$.

where $I_{m}^{U}, I_{m}^{L}$ are the upper and lower triangular matrix, respectively.

Proof. It is straightforward.

Analogously, the equivalence relations $\simeq_{e_{t}, \alpha}^{\tau}$ and $\simeq_{e_{t}}^{\tau, \beta}$ can be applied to compute the following two square matrices

$E_{\alpha}^{U}\left(e_{t}\right)=\left[e_{\alpha}^{u}\left(e_{t}\right)_{i j}\right]_{m \times m}$ and $E_{\beta}^{L}\left(e_{t}\right)=\left[e_{\beta}^{l}\left(e_{t}\right)_{i j}\right]_{m \times m}$, respectively, where $\alpha=\left[\alpha_{1}, \alpha_{2}\right]$, $\beta=\left[\beta_{1}, \beta_{2}\right] \subseteq \mathbb{I}$.

Definition 6. Consider the binary relations $\simeq_{e_{t}, \alpha}^{\tau}$ and $\simeq_{e_{t}}^{\tau, \beta}$ and threshold intervals $\alpha=\left[\alpha_{1}, \alpha_{2}\right]$, $\beta=\left[\beta_{1}, \beta_{2} \subseteq \mathbb{I}\right.$. We define

$$
E_{\alpha}^{U}\left(e_{t}\right)=\left[e_{\alpha}^{u}\left(e_{t}\right)_{i j}\right]_{m \times m}: e_{\alpha}^{u}\left(e_{t}\right)_{i j}=\left\{\begin{array}{cc}
1 & \text { if } x_{i} \simeq_{e_{t}, \alpha}^{\tau} x_{j} \\
0 & \text { otherwis }
\end{array}\right.
$$

and

$$
E_{\beta}^{L}\left(e_{t}\right)=\left[e_{\beta}^{l}\left(e_{t}\right)_{i j}\right]_{m \times m}: e_{\beta}^{l}\left(e_{t}\right)_{i j}=\left\{\begin{array}{cc}
1 & \text { if } x_{i} \simeq_{e_{t}, \beta}^{\tau} x_{j} \\
0 & \text { otherwis }
\end{array}\right.
$$

Proposition 5. Let $(X, E, \tau)$ be an IVFST and $E_{\alpha}^{U}\left(e_{t}\right)$ and $E_{\beta}^{L}\left(e_{t}\right)$ be the comparison matrices defined in Equations (5) and (6). Then,

1. For any $1 \leq i \leq m: e_{\alpha}^{u}\left(e_{t}\right)_{i i}=1$ and $e_{\beta}^{l}\left(e_{t}\right)_{i i}=1$.

2. $E^{U}\left(e_{t}\right)$ and $E^{L}\left(e_{t}\right)$ are symmetric matrices.

3. If $e_{\alpha}^{u}\left(e_{t}\right)_{i k}=e_{\alpha}^{u}\left(e_{t}\right)_{j k}=1$, then $e_{\alpha}^{u}\left(e_{t}\right)_{i j}=e_{\alpha}^{u}\left(e_{t}\right)_{j i}=1$. If $e_{\beta}^{l}\left(e_{t}\right)_{i k}=e_{\beta}^{l}\left(e_{t}\right)_{j k}=1$, then $e_{\beta}^{l}\left(e_{t}\right)_{i j}=e_{\beta}^{l}\left(e_{t}\right)_{j i}=1$.

4. If $e_{\alpha}^{u}\left(e_{t}\right)_{k i}=e_{\alpha}^{u}\left(e_{t}\right)_{k j}=1$, then $e_{\alpha}^{u}\left(e_{t}\right)_{i j}=e_{\alpha}^{u}\left(e_{t}\right)_{j i}=1$. If $e_{\beta}^{l}\left(e_{t}\right)_{k i}=e_{\beta}^{l}\left(e_{t}\right)_{k j}=1$, then $e_{\beta}^{l}\left(e_{t}\right)_{i j}=e_{\beta}^{l}\left(e_{t}\right)_{j i}=1$.

where $i, j, k \in\{1, \cdots, m\}$

Proof. It is straightforward.

\section{An Application in Decision-Making Problems}

The main task in decision making methods is to rank the given candidates to find the optimum choice. Since the proposed preorderings, given in Section 3, are not total or linear, we define a score function $S$ based on the entries of defined comparison matrices to obtain a new ranking system of objects according to preorderings $\succsim_{e_{t}, \alpha}^{\tau}$ and $\precsim_{e_{t}}^{\tau, \beta}$.

Definition 7. Let $X$ and $E$ be the universal sets of objects and parameters, respectively, and $\alpha, \beta \subseteq$ $\mathbb{I}$, where $\alpha=\left[\alpha_{1}, \alpha_{1}\right]$ and $\beta=\left[\beta_{1}, \beta_{2}\right]$, are the threshold intervals. The mapping $S=X \rightarrow \mathbb{R}$ defined by

$$
S\left(x_{i}\right)=S_{i}=\sum_{t=1}^{n}\left(\left[\sum_{j=1}^{m} g_{\alpha}\left(e_{t}\right)_{i j}-\sum_{j=1}^{m} e_{\alpha}^{u}\left(e_{t}\right)_{i j}\right]-\left[\sum_{j=1}^{m} s_{\beta}\left(e_{t}\right)_{i j}-\sum_{j=1}^{m} e_{\beta}^{l}\left(e_{t}\right)_{i j}\right]\right)
$$

where $x_{i} \in X$ and $S_{i}$ is score value of object $x_{i}$. 
Example 1. Suppose that $X=\left\{o_{1}, o_{2}, o_{3}, o_{5}\right\}$ be a set of 5 hotels in Langkawi and $E=\left\{e_{1}, \ldots, e_{4}\right\}$ be a set of parameters where for any $t=1, \ldots, 4$ the parameter $e_{t}$ stands for "location", "cleanliness", "facilities", and "food", respectively. Reviewers are classified into three groups: couples, solo travelers, and a group of friends. We consider these groups of reviewers as three different decision-makers, $f_{1}, f_{2}, f_{3}$, characterized based on the criteria $e_{t} \in E$. These three groups provide the following three IVFS matrices $f_{1 E}, f_{2 E}, f_{3 E}$.

Step 1 . The following three interval-valued fuzzy soft set $f_{s E}(s=1,2,3)$ that are given in Tables 1-3.

Table 1. $f_{1 E}$.

\begin{tabular}{cccccc}
\hline$f_{1 E}$ & $\boldsymbol{o}_{\mathbf{1}}$ & $\boldsymbol{o}_{\mathbf{2}}$ & $\boldsymbol{o}_{\mathbf{3}}$ & $\boldsymbol{o}_{\mathbf{4}}$ & $\boldsymbol{o}_{\mathbf{5}}$ \\
\hline$e_{1}$ & {$[0.1,0.4]$} & {$[0.4,0.4]$} & {$[0.4,0.5]$} & {$[0,0.5]$} & {$[0.0,0.0]$} \\
$e_{2}$ & {$[0.5,0.6]$} & {$[0.3,0.6]$} & {$[0.3,1.0]$} & {$[0.7,1.0]$} & {$[0.0,0.7]$} \\
$e_{3}$ & {$[0.0,0.5]$} & {$[0.5,0.8]$} & {$[0.1,0.8]$} & {$[0.1,0.9]$} & {$[0.3,0.9]$} \\
$e_{4}$ & {$[0.0,0.8]$} & {$[0.7,0.8]$} & {$[0.1,0.7]$} & {$[0.1,1.0]$} & {$[0.6,1.0]$} \\
\hline
\end{tabular}

Table 2. $f_{2 E}$.

\begin{tabular}{cccccc}
\hline$f_{2 E}$ & $\boldsymbol{o}_{\mathbf{1}}$ & $\boldsymbol{o}_{\mathbf{2}}$ & $\boldsymbol{o}_{\mathbf{3}}$ & $\boldsymbol{o}_{\mathbf{4}}$ & $\boldsymbol{o}_{\mathbf{5}}$ \\
\hline$e_{1}$ & {$[0.2,0.6]$} & {$[0.2,0.6]$} & {$[0.6,0.6]$} & {$[0.5,0.6]$} & {$[0.5,0.6]$} \\
$e_{2}$ & {$[0.4,0.8]$} & {$[0.0,0.8]$} & {$[0.0,0.6]$} & {$[0.6,0.9]$} & {$[0.6,0.9]$} \\
$e_{3}$ & {$[0.1,0.5]$} & {$[0.1,0.8]$} & {$[0.6,0.8]$} & {$[0.5,0.6]$} & {$[0.5,0.9]$} \\
$e_{4}$ & {$[0.3,0.6]$} & {$[0.3,0.3]$} & {$[0.2,0.3]$} & {$[0.2,0.7]$} & {$[0.7,0.7]$} \\
\hline
\end{tabular}

Table 3. $f_{3 E}$.

\begin{tabular}{cccccc}
\hline$f_{3 E}$ & $\boldsymbol{o}_{\mathbf{1}}$ & $\boldsymbol{o}_{\mathbf{2}}$ & $\boldsymbol{o}_{\mathbf{3}}$ & $\boldsymbol{o}_{\mathbf{4}}$ & $\boldsymbol{o}_{\mathbf{5}}$ \\
\hline$e_{1}$ & {$[0.5,0.6]$} & {$[0.6,0.5]$} & {$[0.1,0.8]$} & {$[0.1,0.5]$} & {$[0.5,0.6]$} \\
$e_{2}$ & {$[0.2,0.8]$} & {$[0.2,0.2]$} & {$[0.2,0.2]$} & {$[0.2,0.6]$} & {$[0.1,0.6]$} \\
$e_{3}$ & {$[0.1,0.3]$} & {$[0.1,0.2]$} & {$[0.2,0.3]$} & {$[0.2,0.3]$} & {$[0.2,0.8]$} \\
$e_{4}$ & {$[0.7,0.8]$} & {$[0.0,0.8]$} & {$[0.0,0.1]$} & {$[0.1,1.0]$} & {$[0.1,0.3]$} \\
\hline
\end{tabular}

Step 2. Assume that $\left[\alpha_{1}, \alpha_{2}\right]=[0.3,0.6]$ and $\left[\beta, \beta_{2}=[0.2,0.4]\right.$.

Step 3. The upper crisp matrices and lower crisp matrices, as below:

$$
\begin{gathered}
f_{1}=\left[\begin{array}{lllll}
0 & 0 & 0 & 0 & 0 \\
0 & 0 & 0 & 1 & 0 \\
0 & 1 & 0 & 0 & 0 \\
0 & 1 & 0 & 0 & 1
\end{array}\right], f_{2}=\left[\begin{array}{lllll}
0 & 0 & 0 & 0 & 0 \\
1 & 0 & 0 & 1 & 1 \\
0 & 0 & 1 & 0 & 1 \\
0 & 0 & 0 & 0 & 1
\end{array}\right], f_{3}=\left[\begin{array}{lllll}
0 & 1 & 0 & 0 & 0 \\
0 & 0 & 0 & 0 & 0 \\
0 & 0 & 0 & 0 & 0 \\
1 & 0 & 0 & 0 & 0
\end{array}\right] \\
f_{1}=\left[\begin{array}{lllll}
0 & 0 & 0 & 0 & 1 \\
0 & 0 & 0 & 0 & 0 \\
0 & 0 & 0 & 0 & 0 \\
0 & 0 & 0 & 0 & 0
\end{array}\right], f_{2}=\left[\begin{array}{lllll}
0 & 0 & 0 & 0 & 0 \\
0 & 0 & 0 & 0 & 0 \\
0 & 0 & 0 & 0 & 0 \\
0 & 0 & 0 & 0 & 0
\end{array}\right], f_{3}=\left[\begin{array}{lllll}
0 & 0 & 0 & 0 & 0 \\
0 & 0 & 0 & 0 & 0 \\
1 & 1 & 0 & 0 & 0 \\
0 & 0 & 1 & 0 & 1
\end{array}\right]
\end{gathered}
$$

Step 4. The upper topology and lower topology are shown in Tables 4 and 5. 
Table 4. $\alpha$-Upper- $e_{t}$ topology; $\alpha=\left[\alpha_{1}, \alpha_{2}\right], t=1, \ldots, 4$.

\begin{tabular}{ccccccccccccc}
\hline \multicolumn{10}{c}{$\tau_{e_{t} \alpha}^{u}$} \\
\hline$e_{1}$ & $\left\{[0]_{1 \times 5}\right.$ & {$[1]_{1 \times 5}$} & {$[0$} & 1 & 0 & 0 & $0]\}$ & & & & & \\
$e_{2}$ & $\left\{[0]_{1 \times 5}\right.$ & {$[1]_{1 \times 5}$} & {$[0$} & 0 & 0 & 1 & $0]$ & {$[1$} & 0 & 0 & 1 & $1]\}$ \\
$e_{3}$ & $\left\{[0]_{1 \times 5}\right.$ & {$[1]_{1 \times 5}$} & {$[0$} & 1 & 0 & 0 & $0]$ & {$[0$} & 0 & 1 & 0 & $1]$ \\
& {$[0$} & 1 & 1 & 0 & $1]\}$ & & & & & & & \\
$e_{4}$ & $\left\{[0]_{1 \times 5}\right.$ & {$[1]_{1 \times 5}$} & {$[0$} & 1 & 0 & 0 & $1]$ & {$[0$} & 0 & 0 & 0 & $1]$ \\
& {$[1$} & 0 & 0 & 0 & $0]$ & {$[1$} & 0 & 0 & 0 & $1]$ & & \\
& {$[1$} & 1 & 0 & 0 & $1]\}$ & & & & & & & \\
\hline
\end{tabular}

Table 5. $\beta$-Lower- $e_{t}$ topology; $\beta=\left[\beta_{1}, \beta_{2}\right], t=1, \ldots, 4$.

\begin{tabular}{cccccccc}
\hline \multicolumn{7}{c}{$\tau_{\boldsymbol{e}_{t}, \beta}^{L}$} \\
\hline$e_{1}$ & $\left\{[0]_{1 \times 5}\right.$ & {$[1]_{1 \times 5}$} & {$[0$} & 0 & 0 & 0 & $1]\}$ \\
$e_{2}$ & $\left\{[0]_{1 \times 5}\right.$ & $\left.[1]_{1 \times 5}\right\}$ & & & & & \\
$e_{3}$ & $\left\{[0]_{1 \times 5}\right.$ & {$[1]_{1 \times 5}$} & {$[1$} & 1 & 0 & 0 & $0]\}$ \\
$e_{4}$ & $\left\{[0]_{1 \times 5}\right.$ & {$[1]_{1 \times 5}$} & {$[0$} & 0 & 1 & 0 & $1]\}$ \\
\hline
\end{tabular}

Step 5. The comparison matrices $G\left(e_{t}, \alpha\right), S\left(e_{t}, \beta\right), E^{U}\left(e_{t}, \alpha\right)$ and $E^{U}\left(e_{t}, \alpha\right)$, over $X$ where $\alpha=\left[\alpha_{1}, \alpha_{2}\right], \beta=\left[\beta_{1}, \beta_{2}\right], t=1, \ldots, 4$ as below:

$$
\begin{aligned}
& G\left(e_{1},[0.3,0.6]\right)=\left[\begin{array}{ccccc}
1 & 0 & 1 & 1 & 1 \\
1 & 1 & 1 & 1 & 1 \\
1 & 0 & 1 & 1 & 1 \\
1 & 0 & 1 & 1 & 1 \\
1 & 0 & 1 & 1 & 1
\end{array}\right] S\left(e_{1}[0.2,0.4)=\left[\begin{array}{lllll}
1 & 1 & 1 & 1 & 1 \\
1 & 1 & 1 & 1 & 1 \\
1 & 1 & 1 & 1 & 1 \\
1 & 1 & 1 & 1 & 1 \\
0 & 0 & 0 & 0 & 1
\end{array}\right]\right. \\
& G\left(e_{2},[0.3,0.6]\right)=\left[\begin{array}{lllll}
1 & 1 & 1 & 0 & 1 \\
0 & 1 & 1 & 0 & 0 \\
0 & 1 & 1 & 0 & 0 \\
1 & 1 & 1 & 1 & 1 \\
1 & 1 & 1 & 1 & 1
\end{array}\right] S\left(e_{2}[0.2,0.4)=\left[\begin{array}{lllll}
1 & 1 & 1 & 1 & 1 \\
1 & 1 & 1 & 1 & 1 \\
1 & 1 & 1 & 1 & 1 \\
1 & 1 & 1 & 1 & 1 \\
1 & 1 & 1 & 1 & 1
\end{array}\right]\right. \\
& G\left(e_{3},[0.3,0.6]\right)=\left[\begin{array}{lllll}
1 & 0 & 0 & 1 & 0 \\
1 & 1 & 0 & 1 & 0 \\
1 & 0 & 1 & 1 & 1 \\
1 & 0 & 0 & 1 & 0 \\
1 & 0 & 1 & 1 & 1
\end{array}\right] S\left(e_{3},[0.2,0.4)=\left[\begin{array}{lllll}
1 & 1 & 0 & 0 & 0 \\
1 & 1 & 0 & 0 & 0 \\
1 & 1 & 1 & 1 & 1 \\
1 & 1 & 1 & 1 & 1 \\
1 & 1 & 1 & 1 & 1
\end{array}\right]\right. \\
& G\left(e_{4},[0.3,0.6]\right)=\left[\begin{array}{lllll}
1 & 0 & 1 & 1 & 0 \\
0 & 1 & 1 & 1 & 0 \\
0 & 0 & 1 & 1 & 0 \\
0 & 0 & 1 & 1 & 0 \\
0 & 1 & 1 & 1 & 1
\end{array}\right] S\left(e_{4},[0.2,0.4)=\left[\begin{array}{ccccc}
1 & 1 & 1 & 1 & 1 \\
1 & 1 & 1 & 1 & 1 \\
0 & 0 & 1 & 0 & 1 \\
1 & 1 & 1 & 1 & 1 \\
0 & 0 & 1 & 0 & 1
\end{array}\right]\right. \\
& E^{U}\left(e_{1},[0.3,0.6]\right)=\left[\begin{array}{ccccc}
1 & 0 & 1 & 1 & 1 \\
0 & 1 & 0 & 0 & 0 \\
1 & 0 & 1 & 1 & 1 \\
1 & 0 & 1 & 1 & 1 \\
1 & 0 & 1 & 1 & 1
\end{array}\right] E^{L}\left(e_{1},[0.2,0.4)=\left[\begin{array}{ccccc}
1 & 1 & 1 & 1 & 0 \\
1 & 1 & 1 & 1 & 0 \\
1 & 1 & 1 & 1 & 0 \\
1 & 1 & 1 & 1 & 0 \\
0 & 0 & 0 & 0 & 1
\end{array}\right]\right.
\end{aligned}
$$




$$
\begin{aligned}
& E^{U}\left(e_{2},[0.3,0.6]\right)=\left[\begin{array}{lllll}
1 & 0 & 0 & 0 & 1 \\
0 & 1 & 1 & 0 & 0 \\
0 & 1 & 1 & 0 & 0 \\
0 & 0 & 0 & 0 & 1 \\
1 & 0 & 0 & 1 & 1
\end{array}\right] E^{L}\left(e_{2},[0.2,0.4)=\left[\begin{array}{lllll}
1 & 1 & 1 & 1 & 1 \\
1 & 1 & 1 & 1 & 1 \\
1 & 1 & 1 & 1 & 1 \\
1 & 1 & 1 & 1 & 1 \\
1 & 1 & 1 & 1 & 1
\end{array}\right]\right. \\
& E^{U}\left(e_{3},[0.3,0.6]\right)=\left[\begin{array}{lllll}
1 & 0 & 0 & 1 & 0 \\
0 & 1 & 0 & 0 & 0 \\
0 & 0 & 1 & 0 & 1 \\
1 & 0 & 0 & 1 & 0 \\
0 & 0 & 1 & 0 & 1
\end{array}\right] E^{L}\left(e_{3},[0.2,0.4)=\left[\begin{array}{lllll}
1 & 1 & 0 & 0 & 0 \\
1 & 1 & 0 & 0 & 0 \\
0 & 0 & 1 & 1 & 1 \\
0 & 0 & 1 & 1 & 1 \\
0 & 0 & 1 & 1 & 1
\end{array}\right]\right. \\
& E^{U}\left(e_{4},[0.3,0.6]\right)=\left[\begin{array}{lllll}
1 & 0 & 0 & 0 & 0 \\
0 & 1 & 0 & 0 & 0 \\
0 & 0 & 1 & 1 & 0 \\
0 & 0 & 1 & 1 & 0 \\
0 & 0 & 0 & 0 & 1
\end{array}\right] E^{L}\left(e_{4},[0.2,0.4)=\left[\begin{array}{lllll}
1 & 1 & 0 & 1 & 0 \\
1 & 1 & 0 & 1 & 0 \\
0 & 0 & 1 & 0 & 1 \\
1 & 1 & 0 & 1 & 0 \\
0 & 0 & 1 & 0 & 1
\end{array}\right]\right.
\end{aligned}
$$

Step 6. By using Definition (2), we have,

$$
\begin{array}{r}
S_{1}=r_{1}\left(e_{1} ;[0.3,0.6],[0.2,0.4]\right)+r_{1}\left(e_{2},[0.3,0.6],[0.2,0.4]\right)+r_{1}\left(e_{3},[0.3,0.6],[0.2,0.4]\right) \\
+r_{1}\left(e_{4},[0.3,0.6],[0.2,0.4]=1 .\right.
\end{array}
$$

Similarly, $S_{2}=5, S_{3}=0, S_{4}=-1, S_{6}=5$.

Step 7 . Then, the ordering is obtained as below

$$
o_{2} \simeq o_{5} \succeq o_{1} \succeq o_{3} \succeq o_{4}
$$

Steps 8 and 9 . Accordingly, $o_{2}$ and $o_{5}$ can be the best objects (Acceptance region), while $o_{4}$ not be selected(Rejection region), and $o_{1}, o_{3}$ cannot be judged(Boundary region).

\subsection{Comparison with Existing Methods}

In this section, we will apply and compare present method and other methods $[25,43,44]$ using real-life example via datasets given in [47] Table 8 from the www.weather.com.cn website. (accessed on 15 May 2021).

Example 2. Let an IFVSs $f_{E}$ describes a family who wants to go to a city in China. Suppose that the weather provides a forecast for fifteen cities in China during the holiday, $X=\left\{o_{1}, \ldots, o_{15}\right\}$, which is shown in Table 6. Suppose that the data of weather forecast describes five parameters $E=\left\{e_{1}, e_{2}, e_{3}, e_{4}, e_{5}\right\}$. Parameters $e_{t}, t=1, \ldots, 5$, stand for "temperature", "air quality index", "levels of ultraviolet radiation", "wind speed", "precipitation", respectively.

Step 1. The IVFSs $f_{E}$ is given in Table 6 .

Step 2. Suppose that

$\alpha=[0.67,0.92],[0.75,0.94],[0.66,0.92],[0.49,0.75],[0.96,0.99]$

$\beta=[0.14,0.8],[0.37,0.77],[0.25,0.76],[0.26,0.76],[0.67,1]$,

where $\alpha=\left[\alpha_{1}, \alpha_{2}\right], \beta=\left[\beta_{1}, \beta_{2}\right]$ 
Table 6. Table for $f_{E}$.

\begin{tabular}{cccccc}
\hline$f_{E}$ & $\boldsymbol{e}_{1}$ & $\boldsymbol{e}_{2}$ & $\boldsymbol{e}_{3}$ & $\boldsymbol{e}_{4}$ & $\boldsymbol{e}_{5}$ \\
\hline$o_{1}$ & {$[0.14,0.86]$} & {$[0.21,0.97]$} & {$[0.0,0.47]$} & {$[0.25,1.0]$} & {$[1.0,1.0]$} \\
$o_{2}$ & {$[0.43,0.82]$} & {$[0.45,0.78]$} & {$[0.0,0.33]$} & {$[0.25,1.0]$} & {$[0.83,1]$} \\
$o_{3}$ & {$[0.64,1.0]$} & {$[0.26,0.63]$} & {$[0.0,0.73]$} & {$[0.5,1.0]$} & {$[1.0,1.0]$} \\
$o_{4}$ & {$[0.5,0.82]$} & {$[0.45,0.82]$} & {$[0.6,0.93]$} & {$[0.0,1.0]$} & {$[0.97,1]$} \\
$o_{5}$ & {$[0.39,0.68]$} & {$[0.79,0.88]$} & {$[0.67,1.0]$} & {$[0.25,1.0]$} & {$[0.83,1]$} \\
$o_{6}$ & {$[0.68,0.93]$} & {$[0.6,0.77]$} & {$[0.6,0.93]$} & {$[0.5,1.0]$} & {$[0.58,1]$} \\
$o_{7}$ & {$[0.36,0.71]$} & {$[0.37,0.96]$} & {$[0.67,0.93]$} & {$[0.0,0.75]$} & {$[0.96,1]$} \\
$o_{8}$ & {$[0.5,0.89]$} & {$[0.76,0.95]$} & {$[0.67,1.0]$} & {$[0.5,1.0]$} & {$[0.89,1]$} \\
$o_{9}$ & {$[0.25,0.71]$} & {$[0.02,1.0]$} & {$[0.67,1.0]$} & {$[0.0,0.75]$} & {$[0.58,1]$} \\
$o_{10}$ & {$[0.0,0.71]$} & {$[0.53,0.92]$} & {$[0.6,0.93]$} & {$[0.5,0.75]$} & {$[1.0,1.0]$} \\
$o_{11}$ & {$[0.0,0.54]$} & {$[0.58,1.0]$} & {$[0.73,1.0]$} & {$[0.0,0.75]$} & {$[0.67,1]$} \\
$o_{12}$ & {$[0.34,0.89]$} & {$[0.0,1.0]$} & {$[0.67,1.0]$} & {$[0.25,0.75]$} & {$[1.0,1.0]$} \\
$o_{13}$ & {$[0.25,0.71]$} & {$[0.58,1.0]$} & {$[0.73,1.0]$} & {$[0.0,0.75]$} & {$[0.67,1]$} \\
$o_{14}$ & {$[0.34,0.89]$} & {$[0.53,0.95]$} & {$[0.67,0.93]$} & {$[0.25,0.75]$} & {$[1.0,1.0]$} \\
$o_{15}$ & {$[0.25,0.71]$} & {$[0.66,0.97]$} & {$[0.6,0.93]$} & {$[0.25,1.0]$} & {$[0.0,1.0]$} \\
\hline
\end{tabular}

Steps 3 and 4. The $\alpha$-Upper- $e_{t}$ Crisp and $\beta$-Lower- $e_{t}$ Crisp; the $\alpha$-Upper- $e_{t}$ Topology and $\beta$-Lower- $e_{t}$ Topology (where $(t=1, \ldots, 5)$ ) as shown in Tables $7-10$.

Table 7. $\alpha$-Upper- $e_{t} ; t=1, \ldots, 5$.

\begin{tabular}{cccccccccccccccc}
\hline \multicolumn{10}{c}{ Upper- $e_{t}$ Crisp } \\
\hline $\mathrm{x}$ & $x_{1}$ & $x_{2}$ & $x_{3}$ & $x_{4}$ & $x_{5}$ & $x_{6}$ & $x_{7}$ & $x_{8}$ & $x_{9}$ & $x_{10}$ & $x_{11}$ & $x_{12}$ & $x_{13}$ & $x_{14}$ & $x_{15}$ \\
$e_{1}$ & {$[0$} & 0 & 0 & 0 & 0 & 1 & 0 & 0 & 0 & 0 & 0 & 0 & 0 & 0 & 0 \\
$e_{2}$ & {$[0$} & 0 & 0 & 0 & 0 & 0 & 0 & 1 & 0 & 0 & 0 & 0 & 0 & 0 & $0]$ \\
$e_{3}$ & {$[0$} & 0 & 0 & 0 & 1 & 1 & 1 & 1 & 1 & 0 & 1 & 1 & 1 & 1 & $0]$ \\
$e_{4}$ & {$[0$} & 0 & 1 & 0 & 0 & 0 & 0 & 1 & 0 & 1 & 0 & 0 & 0 & 0 & 0 \\
$e_{5}$ & {$[1$} & 0 & 1 & 1 & 0 & 0 & 0 & 0 & 0 & 1 & 0 & 1 & 0 & 1 & $0]$ \\
\hline
\end{tabular}

Table 8. $\beta$-Lower- $e_{t} ; t=1, \ldots, 5$.

\begin{tabular}{cccccccccccccccc}
\hline \multicolumn{10}{c}{ Lower $e_{t}$ Crisp } \\
\hline $\mathrm{x}$ & $x_{1}$ & $x_{2}$ & $x_{3}$ & $x_{4}$ & $x_{5}$ & $x_{6}$ & $x_{7}$ & $x_{8}$ & $x_{9}$ & $x_{10}$ & $x_{11}$ & $x_{12}$ & $x_{13}$ & $x_{14}$ & $x_{15}$ \\
$e_{1}$ & {$[0$} & 0 & 0 & 0 & 0 & 0 & 0 & 0 & 0 & 1 & 1 & 0 & 0 & 0 & 0 \\
$e_{2}$ & {$[0$} & 0 & 1 & 0 & 0 & 0 & 0 & 0 & 0 & 0 & 0 & 0 & 0 & 0 & 0 \\
$e_{3}$ & {$[1$} & 1 & 1 & 0 & 0 & 0 & 0 & 0 & 0 & 0 & 0 & 0 & 0 & 0 & $0]$ \\
$e_{4}$ & {$[0$} & 0 & 0 & 0 & 0 & 0 & 1 & 0 & 1 & 0 & 1 & 1 & 1 & 1 & $0]$ \\
$e_{5}$ & {$[0$} & 0 & 0 & 0 & 0 & 1 & 0 & 0 & 1 & 0 & 0 & 0 & 0 & 0 & 0 \\
\hline
\end{tabular}

Table 9. $\alpha$-Upper- $e_{t}$ topology; $t=1, \ldots, 5$.

\begin{tabular}{|c|c|c|c|c|c|c|c|c|c|c|c|c|c|c|c|c|c|}
\hline & & $\tau_{e_{t, \alpha}}^{u}$ & & & & & & & & & & & & & & & \\
\hline$e_{1}$ & $\left\{[0]_{1 \times 15}\right.$, & {$[1]_{1 \times 15}$} & {$[0$} & 0 & 0 & 0 & 0 & 1 & 0 & 0 & 0 & 0 & 0 & 0 & 0 & 0 & $0]\}$ \\
\hline$e_{2}$ & $\left\{[0]_{1 \times 15}\right.$, & {$[1]_{1 \times 15}$} & {$[0$} & 0 & 0 & 0 & 0 & 0 & 0 & 1 & 0 & 0 & 0 & 0 & 0 & 0 & $0]\}$ \\
\hline$e_{3}$ & $\left\{[0]_{1 \times 15}\right.$, & {$[1]_{1 \times 15}$} & {$[0$} & 0 & 0 & 0 & 1 & 1 & 1 & 1 & 1 & 0 & 1 & 1 & 1 & 1 & $0]\}$ \\
\hline$e_{4}$ & $\left\{[0]_{1 \times 15}\right.$, & {$[1]_{1 \times 15}$} & {$[0$} & 0 & 1 & 0 & 0 & 0 & 0 & 1 & 0 & 1 & 0 & 0 & 0 & 0 & $0]\}$ \\
\hline$e_{5}$ & $\left\{[0]_{1 \times 15}\right.$, & {$[1]_{1 \times 15}$} & {$[1$} & 0 & 1 & 1 & 0 & 0 & 0 & 0 & 0 & 1 & 0 & 1 & 0 & 1 & $0]\}$ \\
\hline
\end{tabular}


Table 10. $\beta$-Lower- $e_{t}$ topology; $t=1, \ldots, 5$.

\begin{tabular}{|c|c|c|c|c|c|c|c|c|c|c|c|c|c|c|c|c|c|}
\hline & & $\tau_{e_{t}, \beta}^{l}$ & & & & & & & & & & & & & & & \\
\hline$e_{1}$ & $\left\{[0]_{1 \times 15}\right.$ & {$[1]_{1 \times 15}$,} & {$[0$} & 0 & 0 & 0 & 0 & 0 & 0 & 0 & 0 & 1 & 1 & 0 & 0 & 0 & $0]\}$ \\
\hline$e_{2}$ & $\left\{[0]_{1 \times 15}\right.$, & {$[1]_{1 \times 15}$,} & {$[0$} & 0 & 1 & 0 & 0 & 0 & 0 & 0 & 0 & 0 & 0 & 0 & 0 & 0 & $0]\}$ \\
\hline$e_{3}$ & $\left\{[0]_{1 \times 15}\right.$, & {$[1]_{1 \times 15}$,} & {$[1$} & 1 & 1 & 0 & 0 & 0 & 0 & 0 & 0 & 0 & 0 & 0 & 0 & 0 & $0]\}$ \\
\hline$e_{4}$ & $\left\{[0]_{1 \times 15}\right.$ & {$[1]_{1 \times 15}$,} & {$[0$} & 0 & 0 & 0 & 0 & 0 & 1 & 0 & 1 & 0 & 1 & 1 & 1 & 1 & $0]\}$ \\
\hline$e_{5}$ & $\left\{[0]_{1 \times 15}\right.$, & {$[1]_{1 \times 15}$,} & {$[0$} & 0 & 0 & 0 & 0 & 1 & 0 & 0 & 1 & 0 & 0 & 0 & 0 & 0 & $0]\}$ \\
\hline
\end{tabular}

Step 5. The comparison matrices $G\left(e_{t}, \alpha\right), S\left(e_{t}, \beta\right), E^{U}\left(e_{t}, \alpha\right)$ and $E^{L}\left(e_{t}, \beta\right)$, where $\alpha=\left[\alpha_{1}, \alpha_{2}\right], \beta=\left[\beta_{1}, \beta_{2}\right], t=1, \ldots, 5$ are below:

$G\left(e_{1},[0.67,0.92]\right)$ and $L\left(e_{1},[0.13,0.8]\right)$

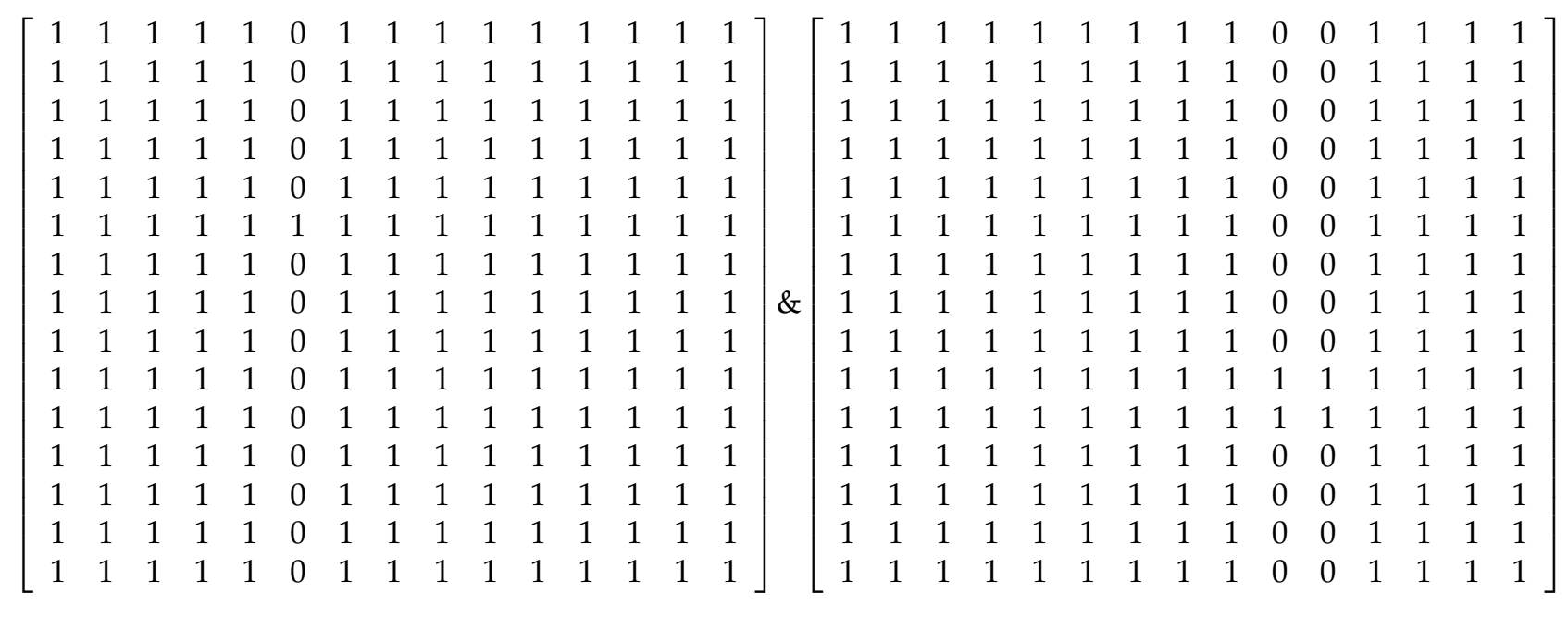

$$
G\left(e_{2},[0.75,0.94]\right) \text { and } L\left(e_{2},[0.37,0.77]\right)
$$

$\left[\begin{array}{lllllllllllllll}1 & 1 & 1 & 1 & 1 & 1 & 1 & 0 & 1 & 1 & 1 & 1 & 1 & 1 & 1 \\ 1 & 1 & 1 & 1 & 1 & 1 & 1 & 0 & 1 & 1 & 1 & 1 & 1 & 1 & 1 \\ 1 & 1 & 1 & 1 & 1 & 1 & 1 & 0 & 1 & 1 & 1 & 1 & 1 & 1 & 1 \\ 1 & 1 & 1 & 1 & 1 & 1 & 1 & 0 & 1 & 1 & 1 & 1 & 1 & 1 & 11 \\ 1 & 1 & 1 & 1 & 1 & 1 & 1 & 0 & 1 & 1 & 1 & 1 & 1 & 1 & 1 \\ 1 & 1 & 1 & 1 & 1 & 1 & 1 & 0 & 1 & 1 & 1 & 1 & 1 & 1 & 1 \\ 1 & 1 & 1 & 1 & 1 & 1 & 1 & 0 & 1 & 1 & 1 & 1 & 1 & 1 & 1 \\ 1 & 1 & 1 & 1 & 1 & 1 & 1 & 1 & 1 & 1 & 1 & 1 & 1 & 1 & 1 \\ 1 & 1 & 1 & 1 & 1 & 1 & 1 & 0 & 1 & 1 & 1 & 1 & 1 & 1 & 1 \\ 1 & 1 & 1 & 1 & 1 & 1 & 1 & 0 & 1 & 1 & 1 & 1 & 1 & 1 & 1 \\ 1 & 1 & 1 & 1 & 1 & 1 & 1 & 0 & 1 & 1 & 1 & 1 & 1 & 1 & 1 \\ 1 & 1 & 1 & 1 & 1 & 1 & 1 & 0 & 1 & 1 & 1 & 1 & 1 & 1 & 1 \\ 1 & 1 & 1 & 1 & 1 & 1 & 1 & 0 & 1 & 1 & 1 & 1 & 1 & 1 & 1 \\ 1 & 1 & 1 & 1 & 1 & 1 & 1 & 0 & 1 & 1 & 1 & 1 & 1 & 1 & 1 \\ 1 & 1 & 1 & 1 & 1 & 1 & 1 & 0 & 1 & 1 & 1 & 1 & 1 & 1 & 1\end{array}\right] \quad \&\left[\begin{array}{llllllllllllllll}1 & 1 & 0 & 1 & 1 & 1 & 1 & 1 & 1 & 1 & 1 & 1 & 1 & 1 & 1 \\ 1 & 1 & 0 & 1 & 1 & 1 & 1 & 1 & 1 & 1 & 1 & 1 & 1 & 1 & 1 \\ 1 & 1 & 1 & 1 & 1 & 1 & 1 & 1 & 1 & 1 & 1 & 1 & 1 & 1 & 1 \\ 1 & 1 & 0 & 1 & 1 & 1 & 1 & 1 & 1 & 1 & 1 & 1 & 1 & 1 & 1 \\ 1 & 1 & 0 & 1 & 1 & 1 & 1 & 1 & 1 & 1 & 1 & 1 & 1 & 1 & 1 \\ 1 & 1 & 0 & 1 & 1 & 1 & 1 & 1 & 1 & 1 & 1 & 1 & 1 & 1 & 1 \\ 1 & 1 & 0 & 1 & 1 & 1 & 1 & 1 & 1 & 1 & 1 & 1 & 1 & 1 & 1 \\ 1 & 1 & 0 & 1 & 1 & 1 & 1 & 1 & 1 & 1 & 1 & 1 & 1 & 1 & 1 \\ 1 & 1 & 0 & 1 & 1 & 1 & 1 & 1 & 1 & 1 & 1 & 1 & 1 & 1 & 1 \\ 1 & 1 & 0 & 1 & 1 & 1 & 1 & 1 & 1 & 1 & 1 & 1 & 1 & 1 & 1 \\ 1 & 1 & 0 & 1 & 1 & 1 & 1 & 1 & 1 & 1 & 1 & 1 & 1 & 1 & 1 \\ 1 & 1 & 0 & 1 & 1 & 1 & 1 & 1 & 1 & 1 & 1 & 1 & 1 & 1 & 1 \\ 1 & 1 & 0 & 1 & 1 & 1 & 1 & 1 & 1 & 1 & 1 & 1 & 1 & 1 & 1 \\ 1 & 1 & 0 & 1 & 1 & 1 & 1 & 1 & 1 & 1 & 1 & 1 & 1 & 1 & 1 \\ 1 & 1 & 0 & 1 & 1 & 1 & 1 & 1 & 1 & 1 & 1 & 1 & 1 & 1 & 1\end{array}\right]$

$G\left(e_{3},[0.66,0.92]\right)$ and $L\left(e_{3},[0.25,0.76]\right)$ 


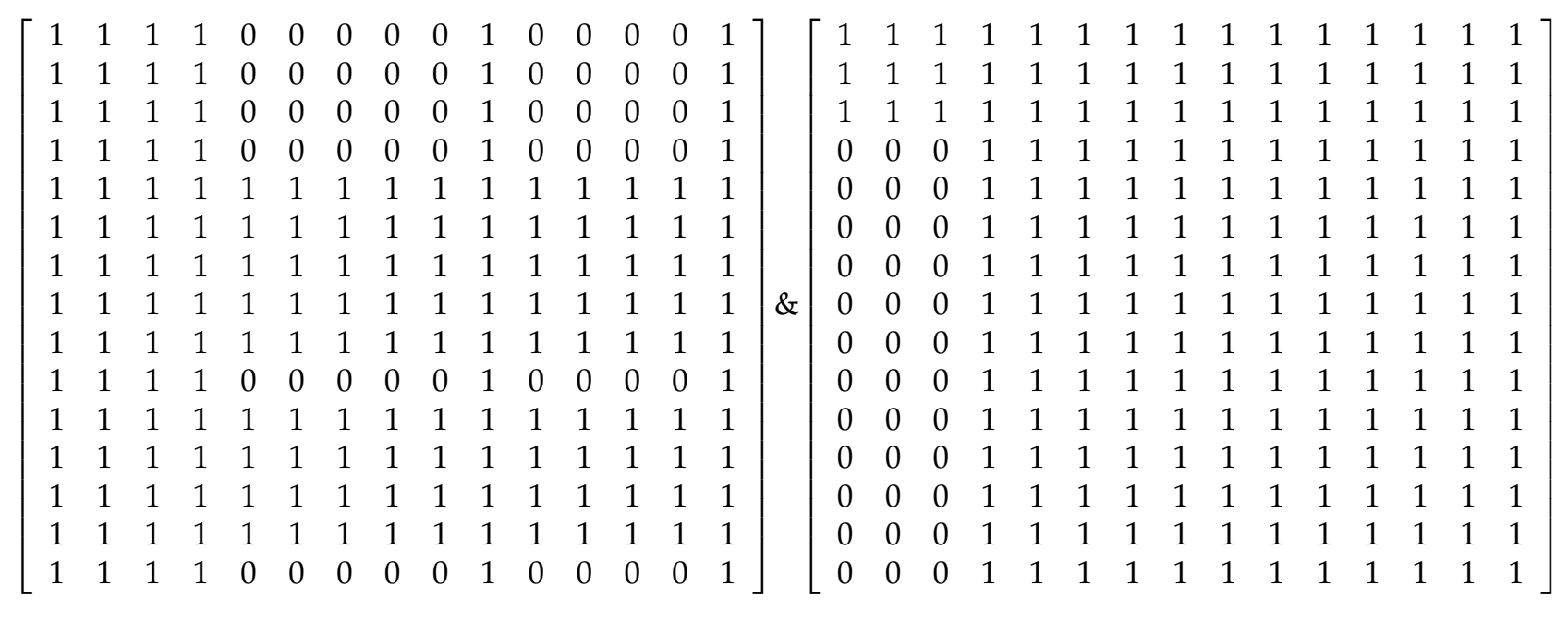

\section{$G\left(e_{4},[0.48,0.74]\right)$ and $L\left(e_{4},[0.26,0.76]\right)$}

$\left[\begin{array}{lllllllllllllll}1 & 1 & 0 & 1 & 1 & 1 & 1 & 0 & 1 & 0 & 1 & 1 & 1 & 1 & 1 \\ 1 & 1 & 0 & 1 & 1 & 1 & 1 & 0 & 1 & 0 & 1 & 1 & 1 & 1 & 1 \\ 1 & 1 & 1 & 1 & 1 & 1 & 1 & 1 & 1 & 1 & 1 & 1 & 1 & 1 & 1 \\ 1 & 1 & 0 & 1 & 1 & 1 & 1 & 0 & 1 & 0 & 1 & 1 & 1 & 1 & 1 \\ 1 & 1 & 0 & 1 & 1 & 1 & 1 & 0 & 1 & 0 & 1 & 1 & 1 & 1 & 1 \\ 1 & 1 & 0 & 1 & 1 & 1 & 1 & 0 & 1 & 0 & 1 & 1 & 1 & 1 & 1 \\ 1 & 1 & 0 & 1 & 1 & 1 & 1 & 0 & 1 & 0 & 1 & 1 & 1 & 1 & 1 \\ 1 & 1 & 1 & 1 & 1 & 1 & 1 & 1 & 1 & 1 & 1 & 1 & 1 & 1 & 1 \\ 1 & 1 & 0 & 1 & 1 & 1 & 1 & 0 & 1 & 0 & 1 & 1 & 1 & 1 & 1 \\ 1 & 1 & 1 & 1 & 1 & 1 & 1 & 1 & 1 & 1 & 1 & 1 & 1 & 1 & 1 \\ 1 & 1 & 0 & 1 & 1 & 1 & 1 & 0 & 1 & 0 & 1 & 1 & 1 & 1 & 1 \\ 1 & 1 & 0 & 1 & 1 & 1 & 1 & 0 & 1 & 0 & 1 & 1 & 1 & 1 & 1 \\ 1 & 1 & 0 & 1 & 1 & 1 & 1 & 0 & 1 & 0 & 1 & 1 & 1 & 1 & 1 \\ 1 & 1 & 0 & 1 & 1 & 1 & 1 & 0 & 1 & 0 & 1 & 1 & 1 & 1 & 1 \\ 1 & 1 & 0 & 1 & 1 & 1 & 1 & 0 & 1 & 0 & 1 & 1 & 1 & 1 & 1\end{array}\right] \&\left[\begin{array}{lllllllllllllll}1 & 1 & 1 & 1 & 1 & 1 & 0 & 1 & 0 & 1 & 0 & 0 & 0 & 0 & 1 \\ 1 & 1 & 1 & 1 & 1 & 1 & 0 & 1 & 0 & 1 & 0 & 0 & 0 & 0 & 1 \\ 1 & 1 & 1 & 1 & 1 & 1 & 0 & 1 & 0 & 1 & 0 & 0 & 0 & 0 & 1 \\ 1 & 1 & 1 & 1 & 1 & 1 & 0 & 1 & 0 & 1 & 0 & 0 & 0 & 0 & 1 \\ 1 & 1 & 1 & 1 & 1 & 1 & 0 & 1 & 0 & 1 & 0 & 0 & 0 & 0 & 1 \\ 1 & 1 & 1 & 1 & 1 & 1 & 0 & 1 & 0 & 1 & 0 & 0 & 0 & 0 & 1 \\ 1 & 1 & 1 & 1 & 1 & 1 & 1 & 1 & 1 & 1 & 1 & 1 & 1 & 1 & 1 \\ 1 & 1 & 1 & 1 & 1 & 1 & 0 & 1 & 0 & 1 & 0 & 0 & 0 & 0 & 1 \\ 1 & 1 & 1 & 1 & 1 & 1 & 1 & 1 & 1 & 1 & 1 & 1 & 1 & 1 & 1 \\ 1 & 1 & 1 & 1 & 1 & 1 & 0 & 1 & 0 & 1 & 0 & 0 & 0 & 0 & 1 \\ 1 & 1 & 1 & 1 & 1 & 1 & 1 & 1 & 1 & 1 & 1 & 1 & 1 & 1 & 1 \\ 1 & 1 & 1 & 1 & 1 & 1 & 1 & 1 & 1 & 1 & 1 & 1 & 1 & 1 & 1 \\ 1 & 1 & 1 & 1 & 1 & 1 & 1 & 1 & 1 & 1 & 1 & 1 & 1 & 1 & 1 \\ 1 & 1 & 1 & 1 & 1 & 1 & 1 & 1 & 1 & 1 & 1 & 1 & 1 & 1 & 1 \\ 1 & 1 & 1 & 1 & 1 & 1 & 0 & 1 & 0 & 1 & 0 & 0 & 0 & 0 & 1\end{array}\right]$

$G\left(e_{5},[0.96,0.99]\right)$ and $L\left(e_{5},[0.67,1]\right)$

$\left[\begin{array}{lllllllllllllll}1 & 1 & 1 & 1 & 1 & 1 & 1 & 1 & 1 & 1 & 1 & 1 & 1 & 1 & 1 \\ 0 & 1 & 0 & 0 & 1 & 1 & 1 & 1 & 1 & 0 & 1 & 0 & 1 & 0 & 1 \\ 1 & 1 & 1 & 1 & 1 & 1 & 1 & 1 & 1 & 1 & 1 & 1 & 1 & 1 & 1 \\ 1 & 1 & 1 & 1 & 1 & 1 & 1 & 1 & 1 & 1 & 1 & 1 & 1 & 1 & 1 \\ 0 & 1 & 0 & 0 & 1 & 1 & 1 & 1 & 1 & 0 & 1 & 0 & 1 & 0 & 1 \\ 0 & 1 & 0 & 0 & 1 & 1 & 1 & 1 & 1 & 0 & 1 & 0 & 1 & 0 & 1 \\ 0 & 1 & 0 & 0 & 1 & 1 & 1 & 1 & 1 & 0 & 1 & 0 & 1 & 0 & 1 \\ 0 & 1 & 0 & 0 & 1 & 1 & 1 & 1 & 1 & 0 & 1 & 0 & 1 & 0 & 1 \\ 0 & 1 & 0 & 0 & 1 & 1 & 1 & 1 & 1 & 0 & 1 & 0 & 1 & 0 & 1 \\ 1 & 1 & 1 & 1 & 1 & 1 & 1 & 1 & 1 & 1 & 1 & 1 & 1 & 1 & 1 \\ 0 & 1 & 0 & 0 & 1 & 1 & 1 & 1 & 1 & 0 & 1 & 0 & 1 & 0 & 1 \\ 1 & 1 & 1 & 1 & 1 & 1 & 1 & 1 & 1 & 1 & 1 & 1 & 1 & 1 & 1 \\ 0 & 1 & 0 & 0 & 1 & 1 & 1 & 1 & 1 & 0 & 1 & 0 & 1 & 0 & 1 \\ 1 & 1 & 1 & 1 & 1 & 1 & 1 & 1 & 1 & 1 & 1 & 1 & 1 & 1 & 1 \\ 0 & 1 & 0 & 0 & 1 & 1 & 1 & 1 & 1 & 0 & 1 & 0 & 1 & 0 & 1\end{array}\right] \&\left[\begin{array}{llllllllllllllll}1 & 1 & 1 & 1 & 1 & 0 & 1 & 1 & 0 & 1 & 1 & 1 & 1 & 1 & 1 \\ 1 & 1 & 1 & 1 & 1 & 0 & 1 & 1 & 0 & 1 & 1 & 1 & 1 & 1 & 1 \\ 1 & 1 & 1 & 1 & 1 & 0 & 1 & 1 & 0 & 1 & 1 & 1 & 1 & 1 & 1 \\ 1 & 1 & 1 & 1 & 1 & 0 & 1 & 1 & 0 & 1 & 1 & 1 & 1 & 1 & 1 \\ 1 & 1 & 1 & 1 & 1 & 0 & 1 & 1 & 0 & 1 & 1 & 1 & 1 & 1 & 1 \\ 1 & 1 & 1 & 1 & 1 & 1 & 1 & 1 & 1 & 1 & 1 & 1 & 1 & 1 & 1 \\ 1 & 1 & 1 & 1 & 1 & 0 & 1 & 1 & 0 & 1 & 1 & 1 & 1 & 1 & 1 \\ 1 & 1 & 1 & 1 & 1 & 0 & 1 & 1 & 0 & 1 & 1 & 1 & 1 & 1 & 1 \\ 1 & 1 & 1 & 1 & 1 & 1 & 1 & 1 & 1 & 1 & 1 & 1 & 1 & 1 & 1 \\ 1 & 1 & 1 & 1 & 1 & 0 & 1 & 1 & 0 & 1 & 1 & 1 & 1 & 1 & 1 \\ 1 & 1 & 1 & 1 & 1 & 0 & 1 & 1 & 0 & 1 & 1 & 1 & 1 & 1 & 1 \\ 1 & 1 & 1 & 1 & 1 & 0 & 1 & 1 & 0 & 1 & 1 & 1 & 1 & 1 & 1 \\ 1 & 1 & 1 & 1 & 1 & 0 & 1 & 1 & 0 & 1 & 1 & 1 & 1 & 1 & 1 \\ 1 & 1 & 1 & 1 & 1 & 0 & 1 & 1 & 0 & 1 & 1 & 1 & 1 & 1 & 1 \\ 1 & 1 & 1 & 1 & 1 & 0 & 1 & 1 & 0 & 1 & 1 & 1 & 1 & 1 & 1\end{array}\right]$

Now, we compute matrices $E^{U}\left(e_{t}, \alpha\right), E^{L}\left(e_{t}, \beta\right) \alpha=\left[\alpha_{1}, \alpha_{2}\right], \beta=\left[\beta_{1}, \beta_{2}\right], t=1, \ldots, 5$ $E^{U}\left(e_{1},[0.67,0.92]\right)$ and $E^{L}\left(e_{1},[0.13,0.8]\right)$ 
$\left[\begin{array}{lllllllllllllll}1 & 1 & 1 & 1 & 1 & 0 & 1 & 1 & 1 & 1 & 1 & 1 & 1 & 1 & 1 \\ 1 & 1 & 1 & 1 & 1 & 0 & 1 & 1 & 1 & 1 & 1 & 1 & 1 & 1 & 1 \\ 1 & 1 & 1 & 1 & 1 & 0 & 1 & 1 & 1 & 1 & 1 & 1 & 1 & 1 & 1 \\ 1 & 1 & 1 & 1 & 1 & 0 & 1 & 1 & 1 & 1 & 1 & 1 & 1 & 1 & 1 \\ 1 & 1 & 1 & 1 & 1 & 0 & 1 & 1 & 1 & 1 & 1 & 1 & 1 & 1 & 1 \\ 0 & 0 & 0 & 0 & 0 & 1 & 0 & 0 & 0 & 0 & 0 & 0 & 0 & 0 & 0 \\ 1 & 1 & 1 & 1 & 1 & 0 & 1 & 1 & 1 & 1 & 1 & 1 & 1 & 1 & 1 \\ 1 & 1 & 1 & 1 & 1 & 0 & 1 & 1 & 1 & 1 & 1 & 1 & 1 & 1 & 1 \\ 1 & 1 & 1 & 1 & 1 & 0 & 1 & 1 & 1 & 1 & 1 & 1 & 1 & 1 & 1 \\ 1 & 1 & 1 & 1 & 1 & 0 & 1 & 1 & 1 & 1 & 1 & 1 & 1 & 1 & 1 \\ 1 & 1 & 1 & 1 & 1 & 0 & 1 & 1 & 1 & 1 & 1 & 1 & 1 & 1 & 1 \\ 1 & 1 & 1 & 1 & 1 & 0 & 1 & 1 & 1 & 1 & 1 & 1 & 1 & 1 & 1 \\ 1 & 1 & 1 & 1 & 1 & 0 & 1 & 1 & 1 & 1 & 1 & 1 & 1 & 1 & 1 \\ 1 & 1 & 1 & 1 & 1 & 0 & 1 & 1 & 1 & 1 & 1 & 1 & 1 & 1 & 1 \\ 1 & 1 & 1 & 1 & 1 & 0 & 1 & 1 & 1 & 1 & 1 & 1 & 1 & 1 & 1\end{array}\right] \quad \&\left[\begin{array}{lllllllllllllll}1 & 1 & 1 & 1 & 1 & 1 & 1 & 1 & 1 & 0 & 0 & 1 & 1 & 1 & 1 \\ 1 & 1 & 1 & 1 & 1 & 1 & 1 & 1 & 1 & 0 & 0 & 1 & 1 & 1 & 1 \\ 1 & 1 & 1 & 1 & 1 & 1 & 1 & 1 & 1 & 0 & 0 & 1 & 1 & 1 & 1 \\ 1 & 1 & 1 & 1 & 1 & 1 & 1 & 1 & 1 & 0 & 0 & 1 & 1 & 1 & 1 \\ 1 & 1 & 1 & 1 & 1 & 1 & 1 & 1 & 1 & 0 & 0 & 1 & 1 & 1 & 1 \\ 1 & 1 & 1 & 1 & 1 & 1 & 1 & 1 & 1 & 0 & 0 & 1 & 1 & 1 & 1 \\ 1 & 1 & 1 & 1 & 1 & 1 & 1 & 1 & 1 & 0 & 0 & 1 & 1 & 1 & 1 \\ 1 & 1 & 1 & 1 & 1 & 1 & 1 & 1 & 1 & 0 & 0 & 1 & 1 & 1 & 1 \\ 1 & 1 & 1 & 1 & 1 & 1 & 1 & 1 & 1 & 0 & 0 & 1 & 1 & 1 & 1 \\ 0 & 0 & 0 & 0 & 0 & 0 & 0 & 0 & 0 & 1 & 1 & 0 & 0 & 0 & 0 \\ 0 & 0 & 0 & 0 & 0 & 0 & 0 & 0 & 0 & 1 & 1 & 0 & 0 & 0 & 0 \\ 1 & 1 & 1 & 1 & 1 & 1 & 1 & 1 & 1 & 0 & 0 & 1 & 1 & 1 & 1 \\ 1 & 1 & 1 & 1 & 1 & 1 & 1 & 1 & 1 & 0 & 0 & 1 & 1 & 1 & 1 \\ 1 & 1 & 1 & 1 & 1 & 1 & 1 & 1 & 1 & 0 & 0 & 1 & 1 & 1 & 1 \\ 1 & 1 & 1 & 1 & 1 & 1 & 1 & 1 & 1 & 0 & 0 & 1 & 1 & 1 & 1\end{array}\right]$

$E^{U}\left(e_{2},[0.75,0.94]\right)$ and $E^{L}\left(e_{2},[0.37,0.77]\right)$

\title{
$E^{U}\left(e_{3},[0.66,0.92]\right)$ and $E^{L}\left(e_{3},[0.25,0.76]\right)$
}

\begin{abstract}
$\left[\begin{array}{lllllllllllllll}1 & 1 & 1 & 1 & 0 & 0 & 0 & 0 & 0 & 1 & 0 & 0 & 0 & 0 & 1 \\ 1 & 1 & 1 & 1 & 0 & 0 & 0 & 0 & 0 & 1 & 0 & 0 & 0 & 0 & 1\end{array}\right]\left[\begin{array}{lllllllllllllll}1 & 1 & 1 & 0 & 0 & 0 & 0 & 0 & 0 & 0 & 0 & 0 & 0 & 0 & 0 \\ 1 & 1 & 1 & 0 & 0 & 0 & 0 & 0 & 0 & 0 & 0 & 0 & 0 & 0 & 0 \\ 1 & 1 & 1 & 0 & 0 & 0 & 0 & 0 & 0 & 0 & 0 & 0 & 0 & 0 & 0\end{array}\right]$ $\begin{array}{lllllllllllllll}1 & 1 & 1 & 1 & 0 & 0 & 0 & 0 & 0 & 1 & 0 & 0 & 0 & 0 & 1\end{array}$ $\begin{array}{lllllllllllllll}1 & 1 & 1 & 1 & 0 & 0 & 0 & 0 & 0 & 1 & 0 & 0 & 0 & 0 & 1\end{array}$ $\begin{array}{lllllllllllllll}0 & 0 & 0 & 0 & 1 & 1 & 1 & 1 & 1 & 0 & 1 & 1 & 1 & 1 & 0\end{array}$ $\begin{array}{lllllllllllllll}0 & 0 & 0 & 0 & 1 & 1 & 1 & 1 & 1 & 0 & 1 & 1 & 1 & 1 & 0\end{array}$ $\begin{array}{lllllllllllllll}0 & 0 & 0 & 0 & 1 & 1 & 1 & 1 & 1 & 0 & 1 & 1 & 1 & 1 & 0\end{array}$ $\begin{array}{lllllllllllllll}0 & 0 & 0 & 0 & 1 & 1 & 1 & 1 & 1 & 0 & 1 & 1 & 1 & 1 & 0\end{array}$ $\begin{array}{lllllllllllllll}0 & 0 & 0 & 0 & 1 & 1 & 1 & 1 & 1 & 0 & 1 & 1 & 1 & 1 & 0 \\ 1 & 1 & 1 & 1 & 0 & 0 & 0 & 0 & 0 & 1 & 0 & 0 & 0 & 0 & 1\end{array}$ $\begin{array}{lllllllllllllll}1 & 1 & 1 & 1 & 0 & 0 & 0 & 0 & 0 & 1 & 0 & 0 & 0 & 0 & 1\end{array}$ $\begin{array}{lllllllllllllll}0 & 0 & 0 & 0 & 1 & 1 & 1 & 1 & 1 & 0 & 1 & 1 & 1 & 1 & 0\end{array}$ $\begin{array}{lllllllllllllll}0 & 0 & 0 & 0 & 1 & 1 & 1 & 1 & 1 & 0 & 1 & 1 & 1 & 1 & 0\end{array}$ $\begin{array}{lllllllllllllll}0 & 0 & 0 & 0 & 1 & 1 & 1 & 1 & 1 & 0 & 1 & 1 & 1 & 1 & 0\end{array}$ $\begin{array}{lllllllllllllll}0 & 0 & 0 & 0 & 1 & 1 & 1 & 1 & 1 & 0 & 1 & 1 & 1 & 1 & 0\end{array}$

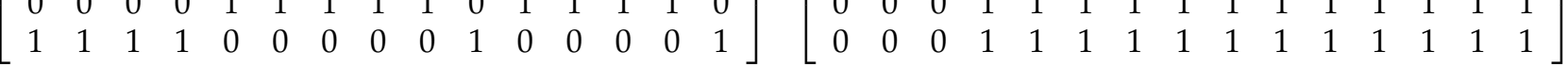
$E^{U}\left(e_{4},[0.48,0.74]\right)$ and $E^{L}\left(e_{4},[0.26,0.76]\right)$
\end{abstract}




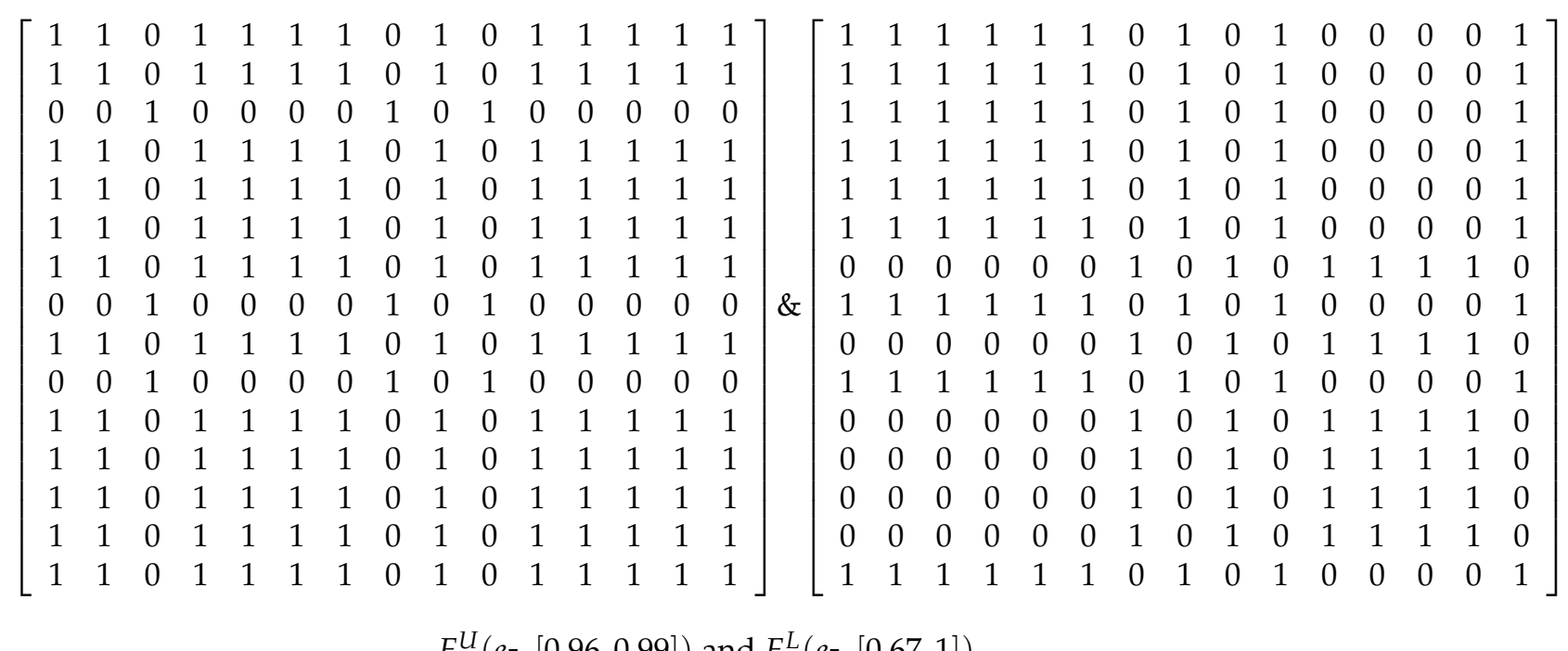

$E^{U}\left(e_{5},[0.96,0.99]\right)$ and $E^{L}\left(e_{5},[0.67,1]\right)$

$\left[\begin{array}{lllllllllllllll}1 & 0 & 1 & 1 & 0 & 0 & 0 & 0 & 0 & 1 & 0 & 1 & 0 & 1 & 0 \\ 0 & 1 & 0 & 0 & 1 & 1 & 1 & 1 & 1 & 0 & 1 & 0 & 1 & 0 & 1 \\ 1 & 0 & 1 & 1 & 0 & 0 & 0 & 0 & 0 & 1 & 0 & 1 & 0 & 1 & 0 \\ 1 & 0 & 1 & 1 & 0 & 0 & - & 0 & 0 & 1 & 0 & 1 & 0 & 1 & 0 \\ 0 & 1 & 0 & 0 & 1 & 1 & 1 & 1 & 1 & 0 & 1 & 0 & 1 & 0 & 1 \\ 0 & 1 & 0 & 0 & 1 & 1 & 1 & 1 & 1 & 0 & 1 & 0 & 1 & 0 & 1 \\ 0 & 1 & 0 & 0 & 1 & 1 & 1 & 1 & 1 & 0 & 1 & 0 & 1 & 0 & 1 \\ 0 & 1 & 0 & 0 & 1 & 1 & 1 & 1 & 1 & 0 & 1 & 0 & 1 & 0 & 1 \\ 0 & 1 & 0 & 0 & 1 & 1 & 1 & 1 & 1 & 0 & 1 & 0 & 1 & 0 & 1 \\ 1 & 0 & 1 & 1 & 0 & 0 & 0 & 0 & 0 & 1 & 0 & 1 & 0 & 1 & 0 \\ 0 & 1 & 0 & 0 & 1 & 1 & 1 & 1 & 1 & 0 & 1 & 0 & 1 & 0 & 1 \\ 1 & 0 & 1 & 1 & 0 & 0 & 0 & 0 & 0 & 1 & 0 & 1 & 0 & 1 & 0 \\ 0 & 1 & 0 & 0 & 1 & 1 & 1 & 1 & 1 & 0 & 1 & 0 & 1 & 0 & 1 \\ 1 & 0 & 1 & 1 & 0 & 0 & 0 & 0 & 0 & 1 & 0 & 1 & 0 & 1 & 0 \\ 0 & 1 & 0 & 0 & 1 & 1 & 1 & 1 & 1 & 0 & 1 & 0 & 1 & 0 & 1\end{array}\right] \&\left[\begin{array}{llllllllllllllll}1 & 1 & 1 & 1 & 1 & 0 & 1 & 1 & 0 & 1 & 1 & 1 & 1 & 1 & 1 \\ 1 & 1 & 1 & 1 & 1 & 0 & 1 & 1 & 0 & 1 & 1 & 1 & 1 & 1 & 1 \\ 1 & 1 & 1 & 1 & 1 & 0 & 1 & 1 & 0 & 1 & 1 & 1 & 1 & 1 & 1 \\ 1 & 1 & 1 & 1 & 1 & 0 & 1 & 1 & 0 & 1 & 1 & 1 & 1 & 1 & 1 \\ 1 & 1 & 1 & 1 & 1 & 0 & 1 & 1 & 0 & 1 & 1 & 1 & 1 & 1 & 1 \\ 0 & 0 & 0 & 0 & 0 & 1 & 0 & 0 & 1 & 0 & 0 & 0 & 0 & 0 & 0 \\ 1 & 1 & 1 & 1 & 1 & 0 & 1 & 1 & 0 & 1 & 1 & 1 & 1 & 1 & 1 \\ 1 & 1 & 1 & 1 & 1 & 0 & 1 & 1 & 0 & 1 & 1 & 1 & 1 & 1 & 1 \\ 0 & 0 & 0 & 0 & 0 & 1 & 0 & 0 & 1 & 0 & 0 & 0 & 0 & 0 & 0 \\ 1 & 1 & 1 & 1 & 1 & 0 & 1 & 1 & 0 & 1 & 1 & 1 & 1 & 1 & 1 \\ 1 & 1 & 1 & 1 & 1 & 0 & 1 & 1 & 0 & 1 & 1 & 1 & 1 & 1 & 1 \\ 1 & 1 & 1 & 1 & 1 & 0 & 1 & 1 & 0 & 1 & 1 & 1 & 1 & 1 & 1 \\ 1 & 1 & 1 & 1 & 1 & 0 & 1 & 1 & 0 & 1 & 1 & 1 & 1 & 1 & 1 \\ 1 & 1 & 1 & 1 & 1 & 0 & 1 & 1 & 0 & 1 & 1 & 1 & 1 & 1 & 1 \\ 1 & 1 & 1 & 1 & 1 & 0 & 1 & 1 & 0 & 1 & 1 & 1 & 1 & 1 & 1\end{array}\right]$

Step 6. By using Definition (7), we have:

$$
\begin{array}{r}
S_{1}=r_{1}\left(e_{1} ;[0.67,0.92],[0.13,0.8]\right)+r_{1}\left(e_{2},[0.75,0.94],[0.37,0.77]\right)+r_{1} \\
\left(e_{3},[0.66,0.92],[0.25,0.76]\right)+r_{1}\left(e_{4},[0.48,0.74],[0.26,0.76]+r_{1}\left(e_{5},[0.96,0.99],[0.67,1]\right.\right. \\
=0+0-3+0+0=-3
\end{array}
$$

Similarly, we have:

$$
\begin{aligned}
& S_{2}=-12, S_{3}=-5, S_{4}=-9, S_{5}=6, S_{6}=7, S_{7}=15, S_{8}=42, S_{9}=2, \\
& S_{8}=42, S_{9}=2, S_{10}=8, S_{11}=-16, S_{12}=6, S_{13}=-3, S_{14}=-9, S_{15}=0 .
\end{aligned}
$$

Step 7. We have the following ordering system on $X$ :

$$
o_{8} \succeq o_{7} \succeq o_{10} \succeq o_{6} \succeq o_{12} \simeq o_{5} \succeq o_{9} \succeq o_{15} \succeq o_{13} \simeq o_{1} \succeq o_{14} \simeq o_{4} \succeq o_{2} \succeq o_{11} .
$$

Steps 8 and 9. Then, from the corresponding object, we obtain, $o_{8}$ to be the best object (Acceptance region), while $o_{11}$ is not selected (Rejection region) and others options $\left(o_{7}, o_{10}, o_{6}, o_{12}, o_{5}, o_{9}, o_{15}\right.$, $\left.o_{13}, o_{1}, o_{14}, o_{4}, o_{2}\right)$ cannot be judged(Boundary region).

Example 3. (Example 2) Let us discuss Example 2 compared to existing methods proposed in $[25,43,44]$ according to the ranking of objects. 
Yang et al. [25] defined the function score value as simply the total of lower and upper membership degrees of objects concerning each parameter. Ma et al. [44] applied Yang's Algorithm 1, which is given in [25] to solve Example 2 and showed the score value as follows: $o_{8} \succeq o_{6} \succeq o_{14} \succeq$ $o_{5} \succeq o_{4} \succeq o_{10} \succeq o_{12} \succeq o_{3} \succeq o_{7} \succeq o_{13} \succeq o_{15} \succeq o_{11} \succeq o_{9} \succeq o_{2} \succeq o_{1}$.

Ma et al. [44] proposed a new efficient decision-making algorithm by using added objects. By using Algorithm 3 Section 4 in [44], Example 2 was solved and the score value for all objects was obtained as follows $o_{8} \succeq o_{6} \succeq o_{14} \succeq o_{5} \succeq o_{4} \succeq o_{10} \succeq o_{12} \succeq o_{3} \succeq o_{7} \succeq o_{13} \succeq o_{15} \succeq o_{11} \succeq$ $o_{9} \succeq o_{2} \succeq o_{1}$.

Ma et al. [43] applied a new decision-making algorithm, based on the average table and the antithesis table - the antithesis the table has symmetry between the objects. Applying Algorithm in [43], Section 3, to solve the Example 2, the following ranking of objects is obtained $o_{8} \succeq o_{6} \succeq$ $o_{5} \succeq o_{14} \succeq o_{4} \succeq o_{12} \succeq o_{10} \succeq o_{3} \succeq o_{13} \succeq o_{7} \succeq o_{15} \succeq o_{11} \succeq o_{2} \succeq o_{9} \succeq o_{1}$.

The comparison results among the present method and methods in $[25,43,44]$ are given in Figure 1.

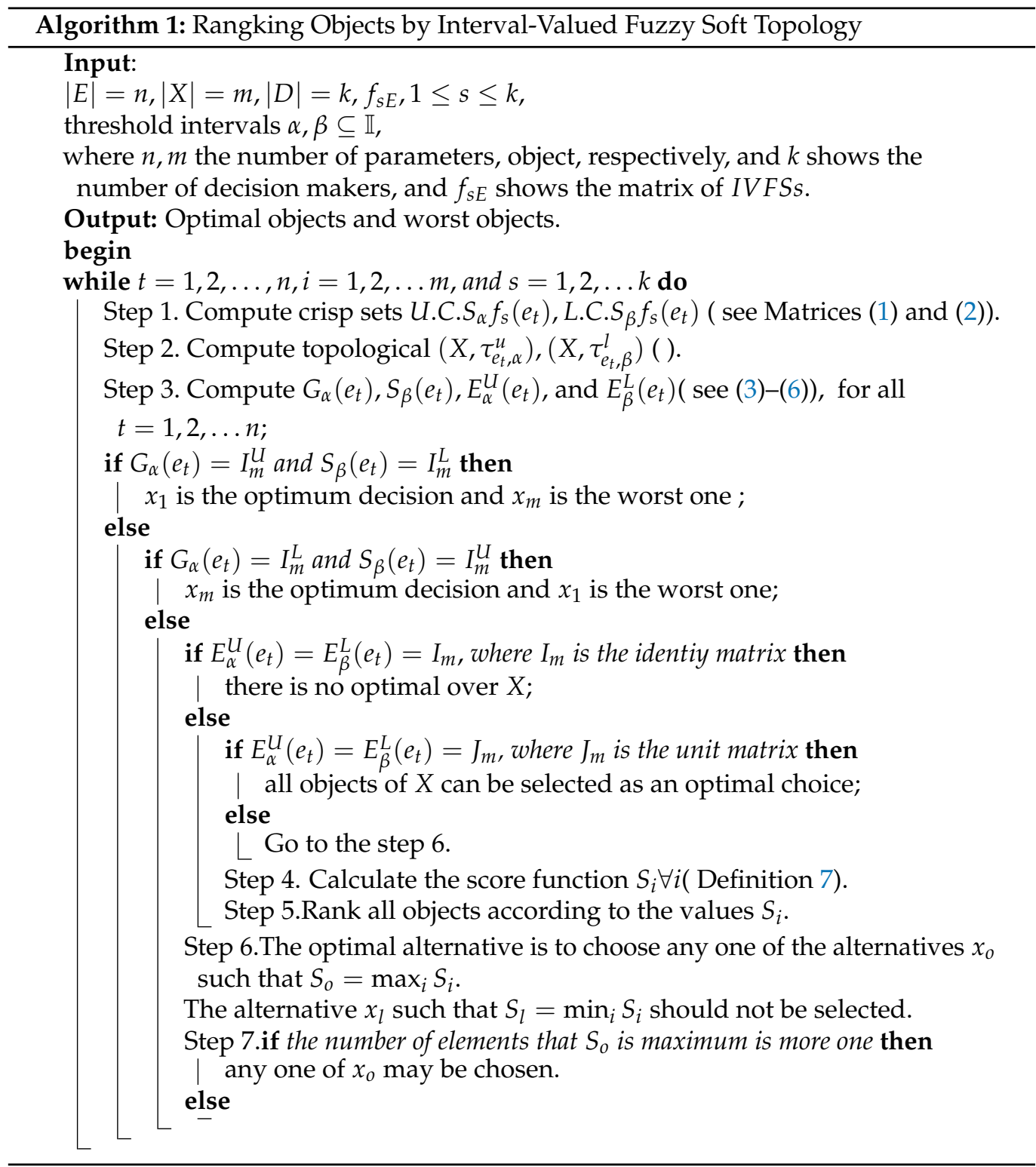




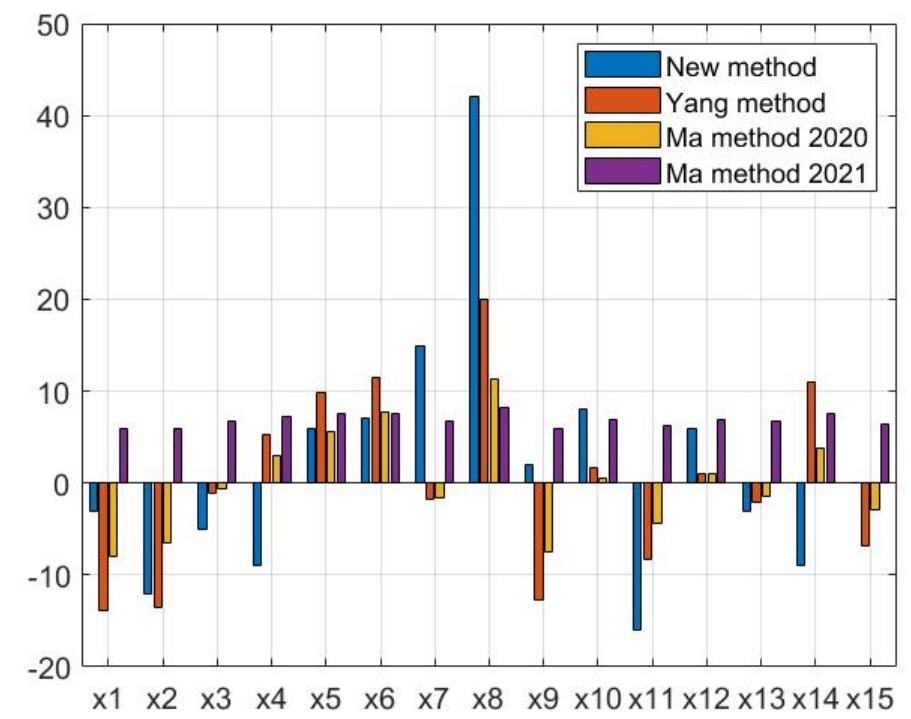

Figure 1. Comparison methods.

\section{Discussion}

According to the present method and the methods proposed in $[25,43,44]$, to reach the process consensus, Yang et al. [25] use the "AND" operator, while methods in [43,44] did not discuss the aggregation problem. In addition, Example 2 shows that all the methods have the same option $o_{8}$, which is the best object. Consequently, algorithms in methods $[25,43,44]$ select just one option, which is the optimum, and do not select the worst option, while the proposed algorithm selects two options-the optimum and as well as the worst option. However, the methods in $[25,43,44]$, rank the objects based on a linear ordering system (see Example 3), while the present method ranks the objects based on preorder relation and a preference relationship, which allows one to have some incomparable objects (nonlinear ordering system). For example, in the Example 2, the objects $o_{12}$ and $o_{5}$ have the same overall score values, which means that these objects cannot be compared with all of the others.

This is the same for the objects $o_{13}, o_{1}$ and $o_{14}, o_{4}$ (see Figure 2). The comparison results between the new proposed method and methods in $[25,43,44]$ are also given in Table 11.

Table 11. Comparison of Existing Methods

\begin{tabular}{lllll}
\hline Methods & $\begin{array}{l}\text { Output } \\
\text { Comparision }\end{array}$ & $\begin{array}{l}\text { Aggregation } \\
\text { Methodology }\end{array}$ & Ranking Methodology & Rank the Objects \\
\hline$[25]$ & optimal option & AND operator & fuzzy choice values & a linear ordering system \\
\hline$[44]$ & optimal option & Not discussed & choice values & a linear ordering system \\
\hline optimal option & Not discussed & $\begin{array}{l}\text { score function computed } \\
\text { from an average and } \\
\text { an antithesis tables }\end{array}$ & a linear ordering system \\
\hline present method & $\begin{array}{l}\text { optimal option } \\
\text { and worst }\end{array}$ & IVFST & $\begin{array}{l}\text { A collective preference } \\
\text { relationship in topological space }\end{array}$ & a nonlinear ordering system \\
\hline
\end{tabular}




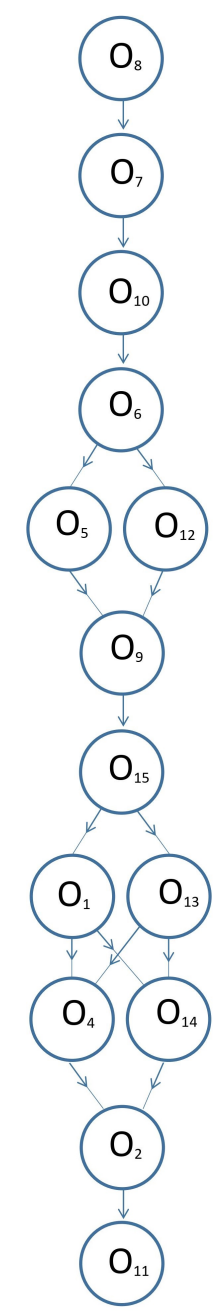

Figure 2. Nonlinear ordering system.

\section{Conclusions}

The interval-valued soft set is a useful tool to deal with fuzziness and uncertainties in decision-making problems. In this paper, we constructed two crisp topological spaces over the set of objects, and then presented two different preorder relations in these topological spaces. By using a new method for ranking data, we proposed an approach for solving multi-attribute group decision-making problems by using a new method for ranking data. Finally, a real-life example has been presented to verify the proposed method approach and to demonstrate the effectiveness by comparing the results with those of some of the existing approaches.

For future research, it would be of merit to apply the decision-making methods into practical applications such as evaluation systems, recommender systems, and conflict handling.

Author Contributions: Formal analysis, A.Z.K.; methodology, A.K. and A.Z.K.; supervision, A.K. and A.Z.K.; validation, A.K.; visualization, A.K.; writing-original draft, M.A.; writing-review \& editing, A.K. All authors contributed equally to the writing of this paper. All authors read and approved the final manuscript.

Funding: The second and third authors gratefully acknowledge the Fundamental Research Grant Schemes, Reference No: FRGS/1/2018/STG06/UPM/01/3, awarded by the Ministry of Higher Education Malaysia.

Institutional Review Board Statement: Not applicable.

Informed Consent Statement: Not applicable. 


\section{Data Availability Statement: Not applicable.}

Acknowledgments: The authors would like to thank the referees and editors for the useful comments and valuable remarks, which improved the current manuscript substantially.

Conflicts of Interest: The authors declare that they have no competing interests.

\section{References}

1. Zadeh, L.A. Fuzzy sets. Inf. Control 1965, 8, 338-353. [CrossRef]

2. Gorzałczany, M.B. A method of inference in approximate reasoning based on interval-valued fuzzy sets. Fuzzy Sets Syst. 1987, 21, 1-17. [CrossRef]

3. Atanassov, K.T. Intuitionistic fuzzy sets. In Intuitionistic Fuzzy Sets; Physica: Heidelberg, Germany, $1999 ;$ pp. 1-137.

4. Pawlak, Z. Rough sets. Int. J. Comput. Inf. Sci. 1982, 11, 341-356. [CrossRef]

5. Molodtsov, D. Soft set theory first results. Comput. Math. Appl. 1999, 37, 19-31 [CrossRef]

6. $\quad$ Maji, P.K.; Biswas, P.; Roy, A.R. A fuzzy soft sets. J. Fuzzy Math. 2001, 9, 89-602.

7. Roy, A.R.; Maji, P.K. A fuzzy soft set theoretic approach to decision making problems. J. Comput. Appl. Math. 2007, 203, 412-418. [CrossRef]

8. Kong, Z.; Gao, L.; Wang, L. Comment on "A fuzzy soft set theoretic approach to decision making problems". J. Comput. Appl. Math. 2009, 223, 540-542. [CrossRef]

9. Feng, F.; Jun, Y.B.; Liu, X.; Li, L. An adjustable approach to fuzzy soft set based decision making. J. Comput. Appl. Math. 2010, 234, 10-20. [CrossRef]

10. Alcantud, J.C.R. A novel algorithm for fuzzy soft set based decision making from multiobserver input parameter data set. Inf. Fusion 2016, 29, 142-148. [CrossRef]

11. Alcantud, J.C.R. Some formal relationships among soft sets, fuzzy sets, and their extensions. Int. J. Approx. Reason. 2016, 68, 45-53. [CrossRef]

12. Alcantud, J.C.R.; Mathew, T.J. Separable fuzzy soft sets and decision making with positive and negative attributes. Appl. Soft Comput. 2017, 59, 586-595. [CrossRef]

13. Aktaş, H.; Çağman, N. Soft decision making methods based on fuzzy sets and soft sets. J. Intell. Fuzzy Syst. 2016, 30, 2797-2803. [CrossRef]

14. Maji, P.K. More on intuitionistic fuzzy soft sets. In International Workshop on Rough Sets, Fuzzy Sets, Data Mining, and Granular-Soft Computing; Springer: Berlin/Heidelberg, Germany, 2009; pp. 231-240.

15. Maji, P.K.; Biswas, R.; Roy, A.R. Intuitionistic fuzzy soft sets. J. Fuzzy Math. 2001, 9, 677-692.

16. Jiang, Y.; Tang, Y.; Chen, Q.; Liu, H.; Tang, J. Interval-valued intuitionistic fuzzy soft sets and their properties. Comput. Math. Appl. 2010, 60, 906-918. [CrossRef]

17. Maji, P K.; Roy, A.R.; Biswas, R. On intuitionistic fuzzy soft sets. J. Fuzzy Math. 2004, 12, 669-684.

18. Liu, Y.; Qin, K.; Martínez, L. Improving decision making approaches based on fuzzy soft sets and rough soft sets. Appl. Soft Comput. 2018, 65, 320-332. [CrossRef]

19. Feng, F.; Li, C.; Davvaz, B.; Ali, M. I. Soft sets combined with fuzzy sets and rough sets: A tentative approach. Soft Comput. 2010, 14, 899-911. [CrossRef]

20. Roy, S.; Samanta, T.K. A note on fuzzy soft topological spaces. Ann. Fuzzy Math. Inform. 2012, 3, $305-311$.

21. Tanay, B.; Kandemir, M.B. Topological structure of fuzzy soft sets. Comput. Math. Appl. 2011, 61, 2952-2957. [CrossRef]

22. Khameneh, A.Z.; Kılıçman, A.; Salleh, A.R. Fuzzy soft boundary. Ann. Fuzzy Math. Inform. 2014, 8, 687-703.

23. Khameneh, A.Z.; Kılıçman, A.; Salleh, A.R. Fuzzy soft product topology. Ann. Fuzzy Math. Inform. $2014,7,935-947$.

24. Son, M.-J. Interval-valued Fuzzy Soft Sets. J. Korean Inst. Intell. Syst. 2007, 17, 557-562. [CrossRef]

25. Yang, X.; Lin, T.Y.; Yang, J.; Li, Y.; Yu, D. Combination of interval-valued fuzzy set and soft set. Comput. Math. Appl. 2009, 58, 521-527. [CrossRef]

26. Feng, F.; Li, Y.; Leoreanu-Fotea, V. Application of level soft sets in decision making based on interval-valued fuzzy soft sets. Comput. Math. Appl. 2010, 60, 1756-1767. [CrossRef]

27. Xiao, Z.; Chen, W.; Li, L. A method based on interval-valued fuzzy soft set for multi-attribute group decision-making problems under uncertain environment. Knowl. Inf. Syst. 2013, 34, 653-669. [CrossRef]

28. Khameneh, A.Z.; Kılıçman, A.; Salleh, A.R. An adjustable approach to multi-criteria group decision-making based on a preference relationship under fuzzy soft information. Int. J. Fuzzy Syst. 2017, 19, 1840-1865. [CrossRef]

29. Khameneh, A.Z.; Kılıçman, A.; Salleh, A.R. Application of a preference relationship in decision-making based on intuitionistic fuzzy soft sets. J. Intell. Fuzzy Syst. 2018, 34, 123-139. [CrossRef]

30. Khameneh, A.Z.; Kılıçman, A.; Salleh, A.R. An adjustable method for data ranking based on fuzzy soft sets. Indian J. Sci. Technol. 2015, 8, 1-9.

31. Jiang, Y.; Tang, Y.; Liu, H.; Chen, Z. Entropy on intuitionistic fuzzy soft sets and on interval-valued fuzzy soft sets. Inf. Sci. 2013, 240, 95-114. [CrossRef]

32. Turksen, I.B.; Zhong, Z. An approximate analogical reasoning schema based on similarity measures and interval-valued fuzzy sets. Fuzzy Sets Syst. 1990, 34, 323-346. [CrossRef]

33. Peng, X.; Yang, Y. Algorithms for interval-valued fuzzy soft sets in stochastic multi-criteria decision making based on regret theory and prospect theory with combined weight. Appl. Soft Comput. 2017, 54, 415-430. [CrossRef] 
34. Peng, X.D.; Yang, Y. Information measures for interval-valued fuzzy soft sets and their clustering algorithm. J. Comput. Appl. 2015, 35, 2350-2354.

35. Chen, W.J.; Zou, Y. Rational decision making models with incomplete information based on interval-valued fuzzy soft sets. J. Comput. 2017, 28, 193-207.

36. Yuan, F.; Hu, M.J. Application of interval-valued fuzzy soft sets in evaluation of teaching quality. J. Hunan Inst. Sci. Technol. 2012, 25, 28-30.

37. Esposito, C.; Moscato, V.; Sperlí, G. Trustworthiness Assessment of Users in Social Reviewing Systems. IEEE Trans. Syst. Man Cybern. Syst. 2021. [CrossRef]

38. Han, Q.; Molinaro, C.; Picariello, A.; Sperli, G.; Subrahmanian, V. S.; Xiong, Y. Generating Fake Documents using Probabilistic Logic Graphs. IEEE Trans. Dependable Secur. Comput. 2021. [CrossRef]

39. Rajarajeswari, P.; Dhanalakshmi, P. Interval-valued fuzzy soft matrix theory. Ann. Pure Appl. Math. 2014, 7, 61-72.

40. Basu, T.M.; Mahapatra, N.K.; Mondal, S.K. Matrices in interval-valued fuzzy soft set theory and their application. S. Asian J. Math. 2014, 4, 1-22.

41. Zhang, Q.; Sun, D. An Improved Decision-Making Approach Based on Interval-valued Fuzzy Soft Set. J. Phys. Conf. Ser. 2021, 1828, 012041. [CrossRef]

42. Ma, X.; Qin, H.; Sulaiman, N.; Herawan, T.; Abawajy, J.H. The parameter reduction of the interval-valued fuzzy soft sets and its related algorithms. IEEE Trans. Fuzzy Syst. 2013, 22, 57-71. [CrossRef]

43. Ma, X.; Wang, Y.; Qin, H.; Wang, J. A Decision-Making Algorithm Based on the Average Table and Antitheses Table for Interval-Valued Fuzzy Soft Set. Symmetry 2020, 12, 1131. [CrossRef]

44. Ma, X.; Fei, Q.; Qin, H.; Li, H.; Chen, W. A new efficient decision making algorithm based on interval-valued fuzzy soft set. Appl. Intell. 2021, 51, 3226-3240. [CrossRef]

45. Peng, X.; Garg, H. Algorithms for interval-valued fuzzy soft sets in emergency decision making based on WDBA and CODAS with new information measure. Comput. Ind. Eng. 2018, 119, 439-452. [CrossRef] [PubMed]

46. Ali, M.; Kılıçman, A.; Khameneh, A.Z. Separation Axioms of Interval-Valued Fuzzy Soft Topology via Quasi-Neighborhood Structure. Mathematics 2020, 8, 178. [CrossRef]

47. Lai, H.; Zhang, D. Fuzzy preorder and fuzzy topology. Fuzzy Sets Syst. 2006, 157, 1865-1885. [CrossRef] 